\title{
Fonctions «spline» et méthode d'éléments finis
}

Revue française d'automatique, informatique, recherche opérationnelle. Analyse numérique, tome 9, no 2 (1975), p. 13-40.

<http://www.numdam.org/item?id=M2AN_1975_9_2_13_0>

(C) AFCET, 1975, tous droits réservés.

L'accès aux archives de la revue "Revue française d'automatique, informatique, recherche opérationnelle. Analyse numérique » implique l'accord avec les conditions générales d'utilisation (http://www.numdam.org/legal. php). Toute utilisation commerciale ou impression systématique est constitutive d'une infraction pénale. Toute copie ou impression de ce fichier doit contenir la présente mention de copyright.

\section{Numdam}

Article numérisé dans le cadre du programme

Numérisation de documents anciens mathématiques

http://www.numdam.org/ 


\title{
FONCTIONS "SPLINE 》 ET METHODE D'ELEMENTS FINIS
}

\author{
par Marc Atteia $\left({ }^{1}\right)$
}

Communiqué par P. G. CIARLET

Résumé. - La méthode des éléments finis est présentée généralement, comme un procédé permettant de générer des bases (d'espaces de Hilbert convenables) particulièrement adaptées à la résolution des problèmes elliptiques par la méthode de Galerkin.

Dans notre article, nous abordons la méthode des éléments finis du point de vue de la topologie algébrique. Nous montrons ainsi que toute fonction d'éléments finis peut être considérée comme une fonction "spline". Notre approche a une portée plus générale qu'il ne paraît dans les exemples classiques que nous avons considérés.

\section{INTRODUCTION}

Le but de cet article est de montrer comment l'on peut identifier une approximation obtenue par une méthode (classique) d'éléments finis à une fonction "spline».

La méthode de base utilisée pour établir cette identité est explicitée dans l'application 3.1. Elle a une portée très générale. Nous nous sommes restreints à l'appliquer aux exemples que nous avons jugé les plus significatifs, pour ne pas allonger démesurément cet article.

Voici, brièvement, comment nous procédons.

Soit $\mathfrak{C}$ un complexe simplicial (géométrique) de $\mathbf{R}^{n}$.

$|\mathfrak{C}|$ désignant le polyèdre associé à $\mathfrak{C}$, considérons une fonction numérique $f$ définie sur $|\mathfrak{G}|$.

(1) U.E.R. de Mathématiques, Université Paul Sabatier, Toulouse.

Revue Française d'Automatique, Informatique et Recherche Opérationnelle $\mathrm{n}^{\circ}$ août 1975, R-2. 
Soit $X$ une partie de $\mathbf{R}^{n}$ dont l'intersection avec $\mathfrak{C}^{0}$ (ensemble des sommets de $\mathscr{C})$ n'est pas vide et $\tau_{f}$ une fonction (classique) d'éléments finis de type « Lagrange " ou « Hermite " interpolant $f$ aux sommets de $\mathscr{C}$ contenus dans $X$. Nous montrons que $\tau_{f}$ coïncide sur $X \cap|\mathscr{G}|$ avec une fonction « spline " $\sigma_{f}$ - qui minimise une fonctionnelle (semi-) hilbertienne sur une variété linéaire (convenable) de $\mathbf{R}^{|\boldsymbol{}|} . \|$

Nous avons rassemblé dans les paragraphes 1 et 2 les résultats sur les noyaux des sous-espaces hilbertiens qui nous sont indispensables et qui sont contenus dans l'article de L. Schwartz et dans le cours de J. Neveu indiqués dans la bibliographie.

Nous exposerons dans un article ultérieur les résultats auxquels la méthode, développée ci-dessous, conduit quand on l'applique aux problèmes de convergence et aux noyaux totalement positifs.

\section{A. NOYAUX DE SOUS-ESPACES HILBERTIENS}

\section{Préliminaires}

(i) Soit $\mathcal{E}$ un espace vectoriel topologique localement convexe séparé et quasi-complet.

Définition 1.1: On dira qu'un sous-espace vectoriel $E$ de $\delta$ est un sousespace admissible de $\mathcal{E}$ si $E$ est muni d'une topologie $\mathfrak{C}_{E}$ localement convexe séparée telle que :

(i) $\left(E, \mathfrak{C}_{E}\right)$ soit quasi-complet

(ii) l'injection canonique $k_{E}$ de $E_{\sigma}$ dans $\&$ soit continue.

On rappelle qu'un sous-espace hilbertien $\mathscr{H}$ de $E$ et un sous-espace vectoriel de $E$, muni d'une structure hilbertienne telle que l'injection canonique $j_{\mathfrak{x}}$ de $\mathcal{T}$ dans $E$ soit continue.

Notons $\chi_{\mathfrak{H}}$ l'isomorphisme canonique de $\overline{\mathscr{H}^{\prime}}$ sur $\mathfrak{H}$.

On a le schéma suivant :

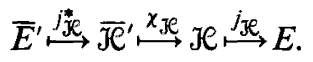

Le noyau de $\mathfrak{H}$ (considéré comme sous-espace hilbertien de $E$ ) est l'élément $H_{E} \in \mathfrak{L}\left(\bar{E}^{\prime}, E_{\sigma}\right)$ défini par $H_{E}=j_{X^{C}} \circ \chi_{\mathcal{H C}^{\circ}} \circ j_{\mathfrak{X}^{*}}$.

Dans la suite, on sera conduit à considérer l'élément $\chi_{\varkappa_{C}} \circ j_{\mathcal{F}} \in \mathfrak{L}\left(\bar{E}_{s}^{\prime}, \mathscr{H}_{\sigma}\right)$ que l'on notera encore $H_{E}$ pour des raisons de commodité. On désignera par Hilb $(E)$ l'ensemble des sous-espaces hilbertiens de $E$.

(ii) $X$ étant un ensemble quelconque, on notera dans la suite, $\mathbf{C}^{X}$ l'espace vectoriel des applications de $X$ dans $\mathbf{C}$, muni de la topologie de la convergence simple. 
Son dual, $\left(\mathbf{C}^{X}\right)^{\prime}$ est l'espace des mesures à support fini dans $X$. Tout élément de $\left(\mathbf{C}^{X}\right)^{\prime}$ est de la forme : $\sum_{x \in N(X)} c_{x} \delta_{x}$ où $N(X)$ est une partie finie de $X, \delta_{x}$ est la mesure de Dirac au point $x \in X$ et $c_{x} \in \mathbf{C}$.

Notons $<,>$ la dualité entre $\mathbf{C}^{X}$ et $\left(\mathbf{C}^{X}\right)^{\prime}$ :

$$
\forall f \in \mathbf{C}^{X},\left\langle f, \sum_{x \in N(X)} c_{x} \delta_{x}\right\rangle=\sum_{x \in N(X)} c_{x} f(x) .
$$

D'autre part, la conjugaison complexe établit sur $\mathbf{C}^{X}\left(\operatorname{resp}\left(\mathbf{C}^{X}\right)^{\prime}\right)$ une anti-involution telle que $\mathbf{C}^{X}$ (resp. $\left.\left(\mathbf{C}^{X}\right)^{\prime}\right)$ puisse être considéré comme son propre anti-espace.

Supposons que $E$ soit un sous-espace admissible de $\mathbf{C}^{\boldsymbol{X}}$. La conjugaison complexe définie sur $\mathbf{C}^{X}$ induit sur $E$ une anti-involution telle que $\bar{E}^{\prime}=E$. Comme $(\bar{E})^{\prime}=\left(\bar{E}^{\prime}\right)$, on en déduit que $\bar{E}^{\prime}=E^{\prime}$.

Soit $k_{E}$ l'injection canonique de $E$ dans $\mathbf{C}^{X}$.

$k_{E}^{*}\left[\left(\mathbf{C}^{X}\right)^{\prime}\right]$ est dense dans $E^{\prime}$ et puisque $\mathbf{C}^{X}$ et $E$ sont leurs propres antiespaces, ${ }^{t} k_{E}\left[\left(\mathbf{C}^{X}\right)^{\prime}\right]$ est dense dans $E^{\prime}$.

Soit $\mathcal{H}$ un sous-espace hilbertien de $E$.

Notons $H(x, t)$ le noyau reproduisant de $\mathscr{H}$ considéré comme sous-espace hilbertien de $\mathbf{C}^{\boldsymbol{X}}$ et posons : $H=\chi_{\mathfrak{F}} \circ j_{\mathfrak{S}}^{*} \circ k_{\boldsymbol{E}}^{*}$.

On sait que :

$$
\forall x \in X, \quad H(., x)=H \delta_{x} \in \mathbf{C}^{X} .
$$

Mais $H \delta_{x}=\left(H_{E} \circ k_{E}^{*}\right) \delta_{x}=H_{E}\left(k_{E}^{*} \cdot \delta_{x}\right) \in E$.

Posons : $\varepsilon_{x}=k_{E}^{*} \delta_{x}, \quad x \in X$.

$\varepsilon_{x}$ est la mesure de Dirac au point $x \in X$, définie sur $E$.

En effet, $\forall x \in X, \forall g \in E$, on a :

$$
\begin{aligned}
g(x)=\left\langle k_{E} \cdot g, \delta_{x}\right\rangle=\left\langle\overline{\bar{k}_{E} \cdot \bar{g}, \bar{\delta}_{x}}\right\rangle & =\left\langle\overline{\bar{k}_{E} \cdot \bar{g}, \delta_{x}}\right\rangle \\
& =\left\langle\bar{g}, k^{*} \delta_{x}\right\rangle_{E, E^{\prime}}=\left\langle g, \bar{\varepsilon}_{x}\right\rangle_{\bar{E}, \bar{E}^{\prime}}=\left\langle g, \bar{\varepsilon}_{x}\right\rangle_{E, E^{\prime \prime}}
\end{aligned}
$$

Mais $\forall x \in E, \forall g \in E$, on a :

$$
\left\langle g, \varepsilon_{x}\right\rangle_{E, E^{\prime}}=\left\langle\overline{\bar{g}, \bar{\varepsilon}_{x}}\right\rangle_{\bar{\varepsilon}, \bar{\varepsilon}^{\prime}}=\left\langle\overline{\bar{g}, \bar{\varepsilon}_{x}}\right\rangle_{E, E^{\prime}}=\overline{\bar{g}(x)}=g(x) .
$$

Donc, $\varepsilon_{x}=\bar{\varepsilon}_{x}$.

EXEMPLE 1.1 :

$$
X=[0,1] \subset \mathbf{R}, E=C^{0}[0,1], \quad \varepsilon=\mathbf{R}^{[0,1]} .
$$

He est l'espace de Hilbert $H^{1}(0,1)$, muni du produit scalaire :

$$
(f \mid g)_{\text {se }}=\int_{0}^{1}\left[f \cdot g+f^{\prime} \cdot g^{\prime}\right](t) \mathrm{d} t
$$

$\mathrm{n}^{\circ}$ août 1975, R-2. 
On a le schéma suivant :

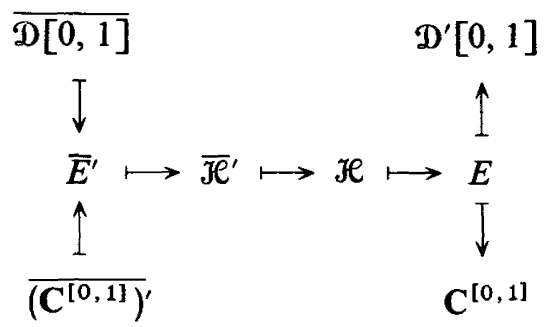

Quelle que soit $\psi \in \mathbb{C}^{\infty}[0,1]$, on a :

$$
(f \mid \psi)_{x e}=\int_{0}^{1}\left[f\left(-\psi^{\prime \prime}+\psi\right)\right](t) \mathrm{d} t+\left(\psi^{\prime} \cdot f\right)_{0}^{1}, \quad f \in H^{1}(0,1) .
$$

On en déduit que le noyau reproduisant de $H^{1}(0,1)$ est la solution $H(x, t)$ du problème différentiel :

$$
\mid \begin{aligned}
& v^{\prime \prime}(t)-v(t)=\delta_{x}(t) \\
& v^{\prime}(0)=v^{\prime}(1)=0
\end{aligned}
$$

On sait que $H(x, t)= \begin{cases}a_{1} e^{x}+a_{2} e^{-x} & \text { si } x \leqslant t \\ b_{1} e_{x}+b_{2} e^{-x} & \text { si } x \geqslant t\end{cases}$

Posons : $c_{i}=b_{i}-a_{i}, i=1,2$.

On a : $\mid \begin{aligned} & c_{1} e^{t}+c_{2} e^{-t}=0 \\ & c_{1} e^{t}-c_{2} e^{-t}=1\end{aligned}$

$$
\Rightarrow c_{1}=\frac{e^{-t}}{2}, \quad c_{2}=-\frac{e^{t}}{2} .
$$

D'autre part, $\begin{aligned} & H_{x}^{\prime}(0, t)=a_{1}-a_{2}=0 \\ & H_{x}^{\prime}(1, t)=b_{1} e-b_{2} e^{-1}=0\end{aligned}$

$$
\frac{e^{t} \cdot e+e^{t} \cdot e^{-1}}{2}+a_{1}\left(e-e^{-1}\right)=0 \Rightarrow a_{1}=-\frac{e^{1-t}+e^{t-1}}{2\left(e-e^{-1}\right)}
$$

$$
\begin{aligned}
b_{1} & =\frac{e^{-t}}{2}-\frac{e^{1-t}+e^{t-1}}{2\left(e-e^{-1}\right)}=\frac{e^{1-t}-e^{-1-t}-e^{1-t}-e^{t-1}}{2\left(e-e^{-1}\right)}=-\frac{e^{t-1}+e^{-t-1}}{2\left(e-e^{-1}\right)} \\
b_{2} & =-\frac{e^{t}}{2}-\frac{e^{1-t}+e^{t-1}}{2\left(e-e^{-1}\right)}=-\frac{e^{1+t}-e^{t-1}+e^{1-t}+e^{t-1}}{2\left(e-e^{-1}\right)} \\
& =-\frac{e^{1-t}+e^{1+t}}{2\left(e-e^{-1}\right)} .
\end{aligned}
$$


D'où :

$$
H(x, t)=\left\{\begin{array}{lll}
\frac{C h x \cdot C h(1-t)}{2 S h t} & \text { si } & 0 \leqslant x \leqslant t \leqslant 1 \\
\frac{C h t \cdot C h(1-x)}{2 S h t} & \text { si } & 0 \leqslant t \leqslant x \leqslant 1
\end{array}\right.
$$

- EXEMPLE 1.2 :

$$
\begin{gathered}
X=[0,1] \subset \mathbf{R}, \quad E=C^{0}[0,1], \quad E=\mathbf{R}^{[0,1]} \\
\mathcal{H}=\left\{f \in \mathbb{D}^{\prime}[0,1] ; f, f^{\prime} \in \mathcal{L}^{2}[0,1] \text { et } f(0)=0\right\} \text { avec } \\
(f \mid g)_{\Re e}=\int_{0}^{1}\left(f^{\prime} \cdot g^{\prime}\right)(t) \mathrm{d} t .
\end{gathered}
$$

Quelle que soit $\psi \in \mathfrak{C}^{\infty}[0,1] \cap \mathcal{H}$, on a :

$$
(f \mid \psi)_{\mathscr{H}}=\int_{0}^{1}\left(-\psi^{\prime \prime} \cdot f\right)(t) \mathrm{d} t+\psi^{\prime}(1) \cdot f(1), \quad f \in \mathcal{H}
$$

On en déduit facilement que :

$$
H(x, t)=-(x-t)_{+}+x=\operatorname{Min}(x, t), \quad x, \quad t \in X
$$

EXEMPLE 1.3 :

$$
\begin{gathered}
X=[0,1] \subset \mathbf{R}, \quad E=C^{1}[0,1], \quad \mathcal{E}=\mathbf{R}^{[0,1]} \\
\mathscr{H}=\left\{f \in \mathfrak{D}^{\prime}[0,1] ; \quad f, f^{\prime}, f^{\prime \prime} \in \mathfrak{L}^{2}[0,1] \text { et } \quad f(0)=f(1)=0\right\} \\
(f \mid g)_{\mathscr{H}}=\int_{0}^{1}\left(f^{\prime \prime} \cdot g^{\prime \prime}\right)(t) \mathrm{d} t .
\end{gathered}
$$

Quelle que soit $\psi \in \mathcal{C}^{\infty}[0,1] \cap \mathcal{H}$, on a :

$$
(f \mid \psi)_{\mathscr{X}}=\int_{0}^{1}\left(-\psi^{(4)} \cdot f\right)(t) \mathrm{d} t+\psi^{\prime \prime}(1) f^{\prime}(1) .
$$

On vérifie facilement que :

$$
H(x, t)=-(x-t)_{+}^{3}-(t-1) x^{3}-\left(t^{3}-3 t^{2}+2 t\right) x, \quad x, t \in X
$$




\section{Opérations sur les noyaux de sous-espaces hilbertiens}

(i) Soient $\mathcal{E}$ et $\mathfrak{F}$ deux espaces vectoriels topologiques, localement convexes séparés et quasi-complets.

Soient d'autre part :

$\left(^{*}\right) E$ un sous-espace admissible de $\mathcal{E}$ et $\mathscr{H}$ un sous-espace hilbertien de $E$, $(* *) u \in \mathfrak{L}\left(\mathcal{E}_{\sigma}, \mathscr{F}_{\sigma}\right)$.

Désignons par $u(E)$ l'image de $E$ par $u$, muni de la topologie localement convexe séparée induite par celle de $\mathscr{F}$, et par $F$, le quasi-complété de $u(E)$.

$F$ est un sous-espace admissible de $\mathcal{F}$.

Notons $u_{E}$ la restriction de $u$ à $E$. On vérifie facilement que $u_{E} \in \mathfrak{L}\left(E_{\sigma}, F_{\sigma}\right)$.

Soit $\mathscr{H}$ un sous-espace hilbertien de $E$ et $u_{\mathcal{H C}}$ la restriction de $u$ à $\mathscr{H}$.

Notons $u(\mathfrak{H C})$ le sous-espace vectoriel de $F$, image de $\mathfrak{H}$ par $u$ et muni de la structure hilbertienne transportée de celle de $\mathscr{X}_{/ \mathrm{Ker}(u)}$ par la bijection $\hat{u}_{\mathcal{F e}}$ de $\mathscr{H}_{/ \operatorname{Ker}(u)} \operatorname{sur} \operatorname{Im}\left(u_{\mathcal{H}}\right)$.

On sait que $u(\mathscr{H}) \in \operatorname{Hilb}(F)$.

Posons $\pi=u(\mathfrak{H})$.

On a le diagramme (commutatif) suivant :

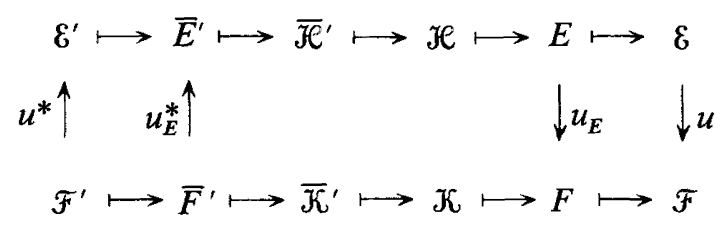

D'où il résulte que :

$$
K_{\mathcal{F}}=u \circ H_{\varepsilon} \circ u^{*} \quad, \quad K_{F}=u_{E} \circ H_{E} \circ u_{E}^{*}
$$

et : $\forall f, g \in \mathscr{H}, \quad(u f \mid u g)_{\varkappa}=(f \mid g)_{\varkappa E}$.

\section{Applications}

Soient $X$ et $Y$ deux ensembles quelconques.

Supposons que $\mathcal{E}=\mathbf{C}^{X}$ et $\mathcal{F}=\mathbf{C}^{X}$.

(*) Multiplication par une fonction

Soit $\alpha$ un élément (quelconque) de $E$.

L'application : $u: \mid \begin{array}{r}\mathbf{C}^{X} \mapsto \mathbf{C}^{X} \\ \mathrm{f} \mapsto \alpha \mathrm{f}\end{array}$ est une application linéaire et continue de $\left(\mathbf{C}^{X}\right)_{\sigma}$ dans $\left(\mathbf{C}^{X}\right)_{\sigma}$.

Revue Française d'Automatique, Informatique et Recherche Opérationnelle 
Posons : $\mathscr{K}=u(\mathscr{H})=\alpha \cdot \mathcal{H}$.

Si $K(x, t)$ désigne le noyau reproduisant de $\pi$, on a :

$$
K(x, t)=\left\langle\left(u \circ H_{\varepsilon} \circ u^{*}\right) \delta_{t}, \delta_{x}\right\rangle .
$$

Mais $\forall f \in \mathcal{E}, \forall x \in X$,

$$
\left\langle f, u^{*} \delta_{x}\right\rangle=\left\langle\bar{u} f, \delta_{x}\right\rangle=\bar{\alpha}(x) \cdot f(x)
$$

et : $\left\langle f,{ }^{t} u \delta_{x}\right\rangle=\alpha(x) \cdot f(x)$.

Il en résulte que :

$$
K(x, t)=\left\langle\left(H_{\varepsilon} \circ u^{*}\right) \delta_{t},{ }^{t} u \delta_{x}\right\rangle=\alpha(x) \cdot \bar{\alpha}(t) \cdot H(x, t)
$$

où $H(x, t)$ est le noyau reproduisant de $\mathcal{H}$.

Le noyau reproduisant de $\alpha \mathcal{H}$ est la fonction :

$$
\mid \begin{aligned}
X \times X & \mapsto \mathbf{C} \\
(x, t) & \mapsto \alpha(x) \cdot \bar{\alpha}(t) \cdot H(x, t)
\end{aligned}
$$

\section{EXEMPLE 2.1:}

Mêmes hypothèses que dans l'exemple 1.2.

Supposons que $\alpha(x)=x^{m}, x \in[0,1]$.

Soit $\mathcal{K}=\alpha \mathcal{H}$. Son noyau est :

$$
K(x, t)=-x^{m} t^{m}\left[(x-t)_{+}-x\right] .
$$

et $\nVdash=\left\{u \in D^{\prime}(0,1) ; u, u^{\prime} \in L^{2}(0,1)\right.$ et $\left.\lim _{x \rightarrow 0-} \frac{u(x)}{x^{m}}=0\right\}$ muni du produit scalaire :

$$
(u \mid v)_{\kappa}=\int_{0}^{1}\left(\frac{u(t)}{t^{m}}\right)^{\prime} \cdot\left(\frac{v(t)}{t^{m}}\right)^{\prime} \mathrm{d} t
$$

(**) Image par une application de $Y$ dans $X$

Soit $\rho$ une application de $Y$ dans $X$.

L'application $u \mid \begin{gathered}\mathbf{C}^{X} \mapsto \mathbf{C}^{\mathbf{Y}} \\ \mathrm{f} \mapsto \mathrm{f} \circ \rho\end{gathered}$ est une application linéaire et continue $\operatorname{de}\left(\mathbf{C}^{\boldsymbol{X}}\right)_{\sigma}$ dans $\left(\mathbf{C}^{\mathbf{Y}}\right)_{\sigma}$.

Posons $\mathcal{K}=u(\mathcal{H})=\mathscr{H} \circ \rho$.

Soit $K(x, t)$ le noyau reproduisant de $\pi$. On a :

$$
K(x, t)=\left\langle\left(\begin{array}{ll}
u \circ H_{\varepsilon} & \left.u^{*}\right) \delta_{t}, \delta_{x}
\end{array}\right\rangle .\right.
$$

$n^{\circ}$ août 1975, R-2. 
Mais $\forall f \in \mathcal{E}, \forall x \in X$,

$$
\left\langle u f, \delta_{x}\right\rangle=f(\rho(x))=\left\langle f,{ }^{t} u \delta_{x}\right\rangle
$$

et

$$
\left\langle f, u^{*} \delta_{x}\right\rangle=\left\langle\bar{u} f, \delta_{x}\right\rangle=\left\langle\overline{u \bar{f}, \delta_{x}}\right\rangle=\overline{\bar{f}}(\rho(x))=f(\rho(x)) .
$$

Il en résulte que :

$$
K(x, t)=\left\langle H_{\varepsilon}\left(u^{*} \delta_{t}\right),{ }^{t} u \delta_{x}\right\rangle=H(\rho(x), \rho(t))
$$

où $H(x, t)$ est le noyau reproduisant de $H$.

Le noyau reproduisant de $\mathfrak{H} \circ \rho$ est la fonction :

$$
\begin{aligned}
& X \times X \mapsto \mathbf{C} \\
& (x, t) \quad \mapsto H(\rho(x), \rho(t)) .
\end{aligned}
$$

\section{EXEMPLE 2.2 :}

$$
X=Y=[0,1] \quad, \quad E=C^{0}(0,1), \quad \mathcal{F}=\mathcal{F}=\mathbf{R}^{[0,1]}
$$

$\mathscr{H}=\left\{u \in \mathfrak{D}^{\prime}(0,1) ; u, u^{\prime} \in L^{2}(0,1)\right.$ et $\left.u(0)=0\right\}$ muni du produit scalaire $(u \mid v)_{1}=\int_{0}^{1} u^{\prime}(t) \cdot v^{\prime}(t) \mathrm{d} t$

$$
\text { Considérons } \rho \mid \begin{aligned}
& X \mapsto Y \\
& x \mapsto x^{m}
\end{aligned}
$$

Soit $\nVdash=\mathscr{H} \circ \rho$. Son noyau est :

$$
K(x, t)=-\left[\left(x^{m}-t^{m}\right)_{+}-x^{m}\right] .
$$

et $\nVdash=\left\{u \in D^{\prime}(0,1) ; u, u^{\prime} \in L^{2}(0,1)\right.$ et $\left.\lim _{x \rightarrow 0-} \frac{u(x)}{x^{m}}=0(1)\right\}$ muni du produit scalaire

$$
(u \mid v)_{\varkappa}=\int_{0}^{1} \frac{u^{\prime}(t) \cdot v^{\prime}(t)}{m t^{m+1}} \mathrm{dt}
$$

(ii) Produit cartésien

Soit $E$ (resp. $F$ ) un sous-espace admissible de $\mathcal{E}($ resp. $\mathcal{F}$ ).

Soit, d'autre part, $\mathscr{H}$ (resp. $\nVdash$ ) un sous-espace hilbertien de $E$ (resp. $F$ ).

Notons $G$ (resp. L) le produit cartésien des espaces topologiques $E$ et $F$ (resp. $\mathcal{H}$ et $\pi$ ). 
On vérifie facilement que $\mathcal{L}$ muni de la norme :

$$
\mid \begin{aligned}
\mathfrak{L} & \mapsto \mathbf{R}_{+} \\
(h, k) & \mapsto\|(h, k)\|_{\mathfrak{L}}=\sqrt{\|h\|_{\mathfrak{K}}^{2}+\|k\|_{\mathfrak{K}}^{2}}
\end{aligned}
$$

est un sous-espace hilbertien de $G$.

Déterminons le noyau $L$ de $\mathcal{L}$ connaissant les noyaux $H$ de $\mathscr{H}$ et $K$ de $\pi$. On sait que :

$$
\begin{array}{r}
\forall l=(h, k) \in \mathfrak{L} \quad, \quad \forall g^{\prime}=\left(e^{\prime}, f^{\prime}\right) \in G^{\prime} \\
\left((h, k) \mid L\left(\bar{e}^{\prime}, \bar{f}^{\prime}\right)\right)_{\mathfrak{L}}=\left(l \mid L \bar{g}^{\prime}\right)_{\mathfrak{L}}=\left\langle l, g^{\prime}\right\rangle=\left\langle(h, k),\left(e^{\prime}, f^{\prime}\right)\right\rangle \\
=\left\langle h, e^{\prime}\right\rangle+\left\langle k, f^{\prime}\right\rangle=\left(h \mid H \bar{e}^{\prime}\right)+\left(k \mid K \bar{f}^{\prime}\right) \\
=\left((h, k) \mid\left(H \bar{e}^{\prime}, K \bar{f}^{\prime}\right)\right) .
\end{array}
$$

Donc :

$$
L\left(e^{\prime}, f^{\prime}\right)=\left(H e^{\prime}, K f^{\prime}\right)
$$

Supposons que $\boldsymbol{E}=\mathbf{C}^{\boldsymbol{X}}, \mathcal{F}=\mathbf{C}^{\boldsymbol{Y}}$.

Notons $H(x, t)$ (resp. $K(y, v), L(x, y ; t, v))$ les noyaux reproduisants de $\mathcal{H}($ resp. $K, \mathcal{L})$. On a :

$$
L(x, y ; t, v)=\left\langle L\left(\delta_{t}, \delta_{v}\right),\left(\delta_{x}, \delta_{y}\right)\right\rangle=\left\langle\left(H \delta_{t}, K \delta_{v}\right),\left(\delta_{x}, \delta_{y}\right)\right\rangle,
$$

c'est-à-dire :

$$
L(x, y ; t, v)=H(x, t)+K(y, v)
$$

EXEMPLE 2.3 :

Supposons que $X=Y=[0,1]$ et que :

$$
E=F=C^{0}[0,1], \quad \varepsilon=\mathbf{R}^{X}, \quad \mathcal{F}=\mathbf{R}^{Y} .
$$

Posons aussi $D=X \times Y$.

Supposons d'autre part que :

$$
\mathscr{H}=\mathcal{K}=\left\{u \in D^{\prime}(0,1) ; u, u^{\prime} \in L^{2}(0,1) \text { et } u(0)=0\right\},
$$

muni du produit scalaire : $(u \mid v)_{x}=\int_{0}^{1} u^{\prime}(t) \cdot v^{\prime}(t) \mathrm{d} t$.

Si $\mathcal{L}=\mathcal{H} \times \mathcal{K}$, on a $: L(x, y ; t, v)=x-(x-t)_{+}+y-(y-v)_{+}$, et $\mathcal{L}=\left\{u \in \mathbb{D}^{\prime}(D) ; \forall(x, y) \in D, u(x, y)=a(x)+b(y)\right.$ avec $a, b, a_{x}^{\prime}, b_{y}^{\prime} \in L^{2}(0,1)$ et $\left.a(0)=b(0)=a^{\prime}(1)=b^{\prime}(1)=0\right\}$ muni du produit scalaire :

$$
(u \mid v)_{\mathfrak{L}}=\int_{0}^{1}\left[a^{\prime}(t) \cdot c^{\prime}(t)+b^{\prime}(t) \cdot d^{\prime}(t)\right] \mathrm{d} t
$$

où $u(x, y)=a(x)+b(y)$ et $v(x, y)=c(x)+d(y)$.

$\mathrm{n}^{\circ}$ août 1975, R-2. 
(iii) Produit tensoriel

$A$ et $B$ étant deux espaces vectoriels topologiques localement convexes, on désignera dans la suite par $A \& B$ (resp. $A \pi B$ ) le produit tensoriel inductif (resp. projectif) de $A$ et $B$.

On notera $A \hat{\varepsilon} B$ (resp. $A \hat{\pi} B$ ) le complété de $A \varepsilon B$ (resp. $A \pi B)$. On sait que si $A$ et $B$ sont séparés, alors $A \varepsilon B$ et $A \hat{\varepsilon} B$ (resp. $A \pi B$ et $A \hat{\pi} B$ ) sont séparés. De plus l'injection canonique $j_{\otimes}$ (resp. $\hat{j}_{\otimes}$ ) de $A \pi B$ dans $A \varepsilon B$ (resp. $A \hat{\pi} B$ dans $A \& B$ ) est continue.

D'autre part $A^{\prime} \otimes B^{\prime}$ est dense dans $(A \hat{\varepsilon} B)_{s}^{\prime}$. (On en déduit que ${ }^{t} \hat{j}_{\otimes}\left(A^{\prime} \otimes B^{\prime}\right)$ est dense dans $(A \hat{\pi} B)_{s}^{\prime}$ puisque ${ }^{t} \hat{j}_{\otimes}(A \hat{\varepsilon} B)_{s}^{\prime}$ est dense dans $\left.(A \hat{\pi} B)_{s}^{\prime}\right)$.

Soit $E$ (resp. $F$ ) un sous-espace admissible de $\mathcal{E}$ (resp. $\mathcal{F}$ ). On vérifie facilement que l'injection de $E \varepsilon F$ (resp. $E \pi F, E \hat{\varepsilon} F, E \hat{\pi} F$ ) dans $\varepsilon \varepsilon \mathcal{F}$ (resp. $\mathcal{E} \pi \mathscr{F}, \mathcal{E} \hat{\varepsilon} \mathcal{F}, \mathcal{E} \hat{\pi} \mathscr{F}$ ) est continue.

Soit $\mathscr{H}$ (resp. $K$ ) un sous-espace hilbertien de $E$ (resp. $F$ ) (de noyau $H_{E}$ (resp. $\left.K_{F}\right)$ ).

Posons : $G_{E \otimes F}=H_{E} \otimes K_{F}$.

Comme $H_{E} \in \mathfrak{L}\left(\bar{E}_{s}^{\prime}, E_{\sigma}\right)$ et $K_{F} \in \mathfrak{L}\left(\bar{F}_{s}^{\prime}, F_{\sigma}\right)$ on sait que : $G_{E \otimes F} \in \mathfrak{L}\left(\bar{E}^{\prime} \varepsilon \bar{F}^{\prime}, E \varepsilon F\right)$. Or $\bar{E}^{\prime} \varepsilon \bar{F}^{\prime}$ est dense dans $(\bar{E} \hat{\varepsilon} \bar{F})_{s}^{\prime}=(\overline{\bar{E} \hat{\varepsilon} F})_{s}^{\prime} . G_{E \otimes F}$ admet donc sur $(\overline{\bar{E} \hat{\varepsilon} \bar{F}})_{s}^{\prime}$ un prolongement $G_{E \varepsilon F} \in \mathfrak{L}\left((\overline{\bar{E} \hat{\varepsilon} F})_{s}^{\prime}, E \varepsilon F\right) \in \mathfrak{L}\left((\overline{\bar{E} \hat{\varepsilon} F})_{s}^{\prime},(E \hat{\varepsilon} F)_{\sigma}\right)$.

De plus, $\forall \eta^{\prime}, \lambda^{\prime} \in E^{\prime}, \forall \xi^{\prime}, \mu^{\prime} \in F^{\prime}$,

$$
\begin{gathered}
\left\langle G_{E \varepsilon F}\left(\bar{\lambda}^{\prime} \otimes \bar{\mu}^{\prime}\right), \eta^{\prime} \otimes \xi^{\prime}\right\rangle=\left\langle G_{E \otimes F}\left(\overline{\lambda^{\prime}} \otimes \bar{\mu}^{\prime}\right), \eta^{\prime} \otimes \xi^{\prime}\right\rangle \\
=\left\langle H_{E} \bar{\lambda}^{\prime}, \eta^{\prime}\right\rangle \cdot\left\langle K_{F} \bar{\mu}^{\prime}, \xi^{\prime}\right\rangle .
\end{gathered}
$$

Il en résulte immédiatement que $G_{E \varepsilon F}$ est un noyau hermitien de type positif. Il définit un sous-espace hilbertien de $E \hat{\varepsilon} F$ qu'on appellera le produit tensoriel de $\mathfrak{H}$ et $\mathcal{K}$ et que l'on notera $\mathcal{H} \hat{\otimes} \mathfrak{K}$.

Supposons que $\varepsilon=\mathbf{C}^{X}, \mathfrak{F}=\mathbf{C}^{Y}(X, Y$ ensembles quelconques). Alors $\mathcal{E} \hat{\varepsilon} \mathfrak{F}=\mathbf{C}^{X \times Y}$.

Notons $H(x, t)$ (resp. $K(y, v), G(z, w))$ les noyaux reproduisants de $\mathscr{H}$ (resp. $\pi, \mathcal{H} \hat{\otimes} \mathcal{K})$.

Si $z=(x, y), w=(t, v)$, alors :

$$
G(z, w)=H(x, t) \cdot K(y, v) .
$$

EXEMPLE 2.4 :

$$
\begin{aligned}
& X=Y=[0,1] \text {. Posons } D=X \times Y . \\
& E=F=C^{0}(0,1) \quad, \quad E \otimes F=C^{0}(D), \quad \varepsilon=\mathbf{R}^{X}, \quad \mathcal{F}=\mathbf{R}^{Y} .
\end{aligned}
$$

Revue Française d'Automatique, Informatique et Recherche Opérationnelle 
$\mathscr{H}=\nVdash=\left\{u \in \mathfrak{D}^{\prime}(0,1) ; u, u^{\prime} \in L^{2}(0,1)\right.$ et $\left.u(0)=0\right\}$, muni du produit scalaire : $(u \mid v)_{1}=\int_{0}^{1} u^{\prime}(t) \cdot v^{\prime}(t) \mathrm{d} t$.

Désignons par $\mathcal{L}$ l'espace $\mathfrak{H} \otimes \nVdash$.

On montre que :

$$
\mathcal{L}=\left\{u \in \mathfrak{D}^{\prime}(D) \quad ; \quad u, u_{x}^{\prime}, u_{y}^{\prime}, u_{x y}^{\prime \prime} \in L^{2}(D)\right.
$$

et

$$
\left.u(0, y)(=u(x, 0))=u_{x}^{\prime}(x, 0)\left(=u_{y}^{\prime}(0, y)\right)=0, x, y \in[0,1]\right\}
$$

muni du produit scalaire :

$$
(u \mid v)_{\mathfrak{E}}=\int_{D} u_{x y}^{\prime \prime}(x, y) \cdot v_{x y}^{\prime \prime}(x, y) \mathrm{d} x \mathrm{~d} y .
$$

\section{(iv) Produits tensoriels symétrique et extérieur}

$A$ étant un espace vectoriel (topologique localement convexe), on désignera dans la suite par $A^{\otimes n}\left(A_{\varepsilon}^{\otimes n}\right), n \in \mathbf{N}^{*}$, le produit tensoriel de $n$ exemplaires de $A$ (muni de la topologie inductive $\varepsilon)(n \geqslant 2)$.

On notera $A^{\vee n}\left(A_{\varepsilon}^{\vee n}\right)$ (resp. $\left.A^{\wedge n}\left(A_{\varepsilon}^{\wedge n}\right)\right)$ le sous-espace vectoriel (topologique) de $A^{\otimes n}\left(A_{\varepsilon}^{\otimes n}\right)$ formé des tenseurs symétriques (resp. antisymétriques).

On notera aussi $\hat{A}_{\varepsilon}^{\vee n}\left(\right.$ resp. $\left.\hat{A}_{\varepsilon}^{\wedge n}\right)$ la fermeture de $A_{\varepsilon}^{\vee n}\left(\right.$ resp. $\left.A^{\wedge n}\right)$ dans $\hat{A}_{\varepsilon}^{\otimes n}$, complété de $A_{\varepsilon}^{\otimes n}$ pour la topologie $\varepsilon$. Si $A$ est séparé, il en est de même de $\hat{A}_{\varepsilon}^{\vee n}$ et $\operatorname{de} \hat{A}_{\varepsilon}^{\wedge n}$.

Etant donné un élément $\sigma$ du nième groupe symétrique $G_{n}$, on désignera par $U_{\sigma}$ l'application :

$$
\mid \begin{aligned}
& A^{\otimes n} \mapsto A^{\otimes n} \\
& a_{1} \otimes \ldots \otimes a_{n} \mapsto a_{\sigma(1)} \otimes \ldots \otimes a_{\sigma(n)}
\end{aligned}
$$

On posera $: u=\frac{1}{n !} \sum_{\sigma \in G_{n}} U_{\sigma}$ (resp. $v=\frac{1}{n !} \sum_{\sigma \in G_{n}} \varepsilon_{\sigma} U_{\sigma}$ où $\varepsilon_{\sigma}$ est la signature de la permutation $\sigma \in G_{n}$ ).

On sait que $u$ (resp. $v$ ) applique $A^{\otimes n} \operatorname{sur} A^{\vee n}\left(\right.$ resp. $\left.A^{\wedge n}\right)$. On vérifie facilement que cette application est faiblement continue de $A_{\varepsilon}^{\otimes n} \operatorname{sur} A_{\varepsilon}^{\vee n}\left(\right.$ resp. $\left.A_{\varepsilon}^{\wedge n}\right)$.

Etant donné $a_{1}, \ldots, a_{n} \in A$, on posera :

$$
a_{1} \vee \ldots \vee a_{n}=u\left(a_{1} \otimes \ldots \otimes a_{n}\right)\left(\text { resp. } a_{1} \wedge \ldots \wedge a_{n}=v\left(a_{1} \otimes \ldots \otimes a_{n}\right)\right) \text {. }
$$

Soit $A^{\prime}$ le dual topologique de $A$. 
Si $a_{1}^{\prime}, \ldots, a_{n}^{\prime} \in A^{\prime}$, on a :

$$
\begin{aligned}
& \left\langle a_{1} \vee \ldots \vee a_{n}, a_{1}^{\prime} \vee \ldots \vee a_{n}^{\prime}\right\rangle=\frac{1}{n !} \sum_{\sigma \in G_{n}}\left\langle a_{1}, a_{\sigma(1)}^{\prime}\right\rangle \ldots\left\langle a_{n}, a_{\sigma(n)}^{\prime}\right\rangle \\
& \left(\operatorname{resp.}\left\langle a_{1} \wedge \ldots \wedge a_{n}, a_{1}^{\prime} \wedge \ldots \wedge a_{n}^{\prime}\right\rangle=\frac{1}{n !} \operatorname{det}\left(\left[\left\langle a_{i}, a_{j}^{\prime}\right\rangle\right]_{1 \leqslant i, j \leqslant n}\right)\right) .
\end{aligned}
$$

Soit $E$ un sous-espace admissible de $\mathcal{E}$ et $\mathscr{H}$ un sous-espace hilbertien de $E$ (de noyau $H_{E}$ ).

On appellera nième puissance tensorielle symétrique (resp. extérieure) de $\mathscr{H}$ et on le désignera par $\mathcal{H}^{\vee n}\left(\right.$ resp. $\left.\mathcal{H}^{\wedge n}\right)$, le sous-espace hilbertien de $\hat{E}_{\varepsilon}^{\vee n}$ (resp. $\hat{E}_{\varepsilon}^{\wedge n}$ ) image de $\mathcal{H}^{\otimes n}$ par l'application $u$ (resp. $v$ ).

Notons $H_{E}^{\vee n}$ (resp. $H_{\hat{E}}^{\hat{E}^{n}}$ ) le noyau de $\mathscr{H}^{\vee n}\left(\right.$ resp. $\mathscr{H}^{\wedge n}$ ) (relativement à $\hat{E}_{\varepsilon}^{\vee n}\left(\right.$ resp. $\left.\left.\hat{E}_{\varepsilon}^{\wedge n}\right)\right)$.

On a : $H_{E}^{n}=u \circ H_{E}^{\otimes n} \circ u^{*}\left(\right.$ resp. $\left.H_{E}^{\vee n}=v \circ H_{E}^{\otimes n} \circ v^{*}\right)$.

On en déduit immédiatement que :

$$
\begin{gathered}
\forall \eta_{1}, \ldots, \eta_{n} \in E, \quad \forall \lambda_{1}^{\prime}, \ldots, \lambda_{n}^{\prime} \in E^{\prime}, \\
\left\langle H_{E}^{\vee n}\left(\bar{\lambda}_{1}^{\prime} \vee \ldots \vee \bar{\lambda}_{1}^{\prime}\right), \eta_{1} \vee \ldots \vee \eta_{n}\right\rangle=\frac{1}{n !} \sum_{\sigma \in G_{n}}\left\langle H_{E} \bar{\lambda}_{1}^{\prime}, \eta_{\sigma(1)}\right\rangle \ldots\left\langle H_{E} \bar{\lambda}_{n}^{\prime}, \eta_{\sigma(n)}\right\rangle \\
\left(\operatorname{resp.}\left\langle H_{E}^{\vee n}\left(\bar{\lambda}_{1}^{\prime} \wedge \ldots \wedge \bar{\lambda}_{n}^{\prime}, \eta_{1} \wedge \ldots \wedge \eta_{n}\right)\right\rangle=\frac{1}{n !} \operatorname{det}\left(\left[\left\langle H_{E} \bar{\lambda}_{i}^{\prime}, \eta_{j}\right\rangle\right]_{1 \leqslant i, j \leqslant n}\right)\right) .
\end{gathered}
$$

Si $\mathcal{E}=\mathbf{C}^{X}, \hat{\boldsymbol{E}}_{\varepsilon}^{\vee n} \subset \mathbf{C}^{X^{n}}$, et l'on aura, avec des notations évidentes :

$$
\begin{aligned}
& H^{\vee n}\left(x_{1}, \ldots, x_{n} ; t_{1}, \ldots, t_{n}\right)=\frac{1}{n !} \sum_{\sigma \in G_{n}} H\left(x_{1}, t_{\sigma(1)}\right) \ldots H\left(x_{n}, t_{\sigma(n)}\right) \\
& \left(\text { resp. } H^{\wedge n}\left(x_{1}, \ldots, x_{n} ; t_{1}, \ldots, t_{n}\right)=\frac{1}{n !} \operatorname{det}\left(\left[H\left(x_{i}, t_{j}\right)\right]_{1 \leqslant i, j \leqslant n}\right) .\right.
\end{aligned}
$$

EXemple 2.5: Supposons que $X=[0,1], E=C^{0}[0,1], \varepsilon=\mathbf{R}^{X}$ et que :

$$
\mathscr{H}=\left\{u \in \mathbb{D}^{\prime}(0,1) ; u, u^{\prime} \in \mathcal{L}^{2}(0,1) \text { et } u(0)=0\right\} .
$$

Soit $\nVdash$ la somme directe hilbertienne des espaces :

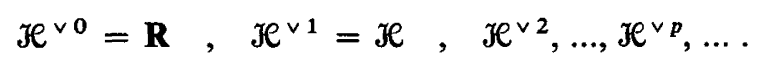

Tout élément $k \in \mathcal{K}$ est une suite $\left(k_{0}, k_{1}, \ldots, k_{p}, \ldots\right)$ et l'on a : $\left(k \mid k^{\prime}\right)_{\aleph_{0}}=\sum_{p \in \mathbb{N}}\left(k_{p} \mid k_{p}^{\prime}\right)_{\mathcal{K}^{v p}}\left(\mathrm{si} k^{\prime}=\left(k_{0}^{\prime}, k_{1}^{\prime}, \ldots, k_{p}^{\prime}, \ldots\right)\right)$.

Etant donné un élément quelconque $h \in \mathfrak{K}$, on vérifie facilement que l'élé$\operatorname{ment}\left(1, h, \frac{h^{\otimes 2}}{\sqrt{2}}, \ldots, \frac{h^{\otimes p}}{\sqrt{p} !}, \ldots\right) \in \pi$ et a pour norme $: e^{\|h\|_{3 e}^{2}}$

Revue Française d'Automatique, Informatique et Recherche Opérationnelle 
Quels que soient $h_{1}, h_{2} \in \mathfrak{H}$, on a :

$$
\left(\operatorname{EXP}\left(h_{1}\right) \mid \operatorname{EXP}\left(h_{2}\right)\right)=e^{\left(h_{1} \mid h_{2}\right)}{ }_{x e} .
$$

D'autre part, la famille $(\operatorname{EXP}(h))_{h \in \mathscr{X}}$ est libre et totale dans $\mathcal{K}$.

$H$ étant le noyau de $\pi$, considérons la fonction $\tilde{K}$ définie sur $X^{2}$ par : $(x, t) \rightarrow \tilde{K}(x ; t)=e^{H(x ; t)} \cdot \tilde{K}$ est un noyau positif sur $X^{2}$ car pour toute suite finie de points distincts $t^{1}, t^{2}, \ldots, t^{n} \in X, n \in \mathrm{N}^{*}$, det $\left(\left[\tilde{K}\left(t^{i} ; t^{j}\right)\right]_{1 \leqslant i, j \leqslant n}\right)$ est un Grammien.

On note que : $\widetilde{K}=\left\{u \in \mathfrak{D}^{\prime}(0,1) ; u, u^{\prime} \in \mathfrak{L}^{2}(0,1)\right\}$ muni du produit scalaire :

$$
(u \mid v)_{\tilde{x}}=u(0) \cdot v(0)+\int_{0}^{1} u^{\prime}(x) \cdot v^{\prime}(x) \cdot \mathrm{e}^{-x} \mathrm{~d} x .
$$

En effet, $\tilde{K}\left(t^{i} ; t^{j}\right)=e^{H\left(t^{i} ; t^{j}\right)}=e^{\left(H_{\left(\cdot ; t^{i}\right) \mid H\left(; t^{j}\right)}\right.}{ }_{\mathcal{H}}=\left(\operatorname{EXP}\left(H\left(. t^{i}\right)\right) \mid \cdot \operatorname{EXP}\left(H\left(. ; t^{j}\right)\right)\right)_{x}$. $\tilde{K}$ définit donc un sous-espace hilbertien $\tilde{\varkappa}$ de $\varepsilon$.

\section{B. FONCTIONS «SPLINE » ET METHODE D'ELEMENTS FINIS}

\section{Fonctions « spline " d'interpolation}

Soient $X$ un ensemble quelconque, $E$ un sous-espace admissible de $\mathbf{C}^{X}$ et $\mathscr{H}$ un sous-espace hilbertien de $E$.

Problème : Etant donné $k_{i} \in \mathscr{H}, \alpha_{i} \in \mathbf{C}, 1 \leqslant i \leqslant n$ et

$$
M_{n}(\alpha)=\left\{h \in \mathcal{H} ;\left(h \mid k_{i}\right)=\alpha_{i}, 1 \leqslant i \leqslant n\right\}
$$

déterminer (s'il existe) l'ensemble :

$$
\Sigma=\left\{\sigma \in M_{n}(\alpha) ; \forall h \in M_{n},\|\sigma\|_{X e} \leqslant\|h\|_{Y C}\right\} .
$$

\section{Résolution du problème}

On sait que si les vecteurs $k_{1}, \ldots, k_{n}$ sont linéairement indépendants, $M_{n}(\alpha)$ n'est pas vide.

Si les vecteurs $k_{1}, \ldots, k_{n}$ sont linéairement dépendants, $M_{n}(\alpha)$ n'est pas vide à condition que $\alpha_{1}, \ldots, \alpha_{n}$ vérifient certaines conditions de compatibilité.

Nous supposerons donc que $k_{1}, \ldots, k_{n}$ sont linéairement indépendants.

$M_{n}(\alpha)$ est alors une variété linéaire fermée de $\mathfrak{H}$. On vérifie sans difficulté que $\Sigma$ se réduit alors à un élément $\sigma$ tel que :

$$
\forall x \in X \quad, \sigma(x)=\sum_{i=1}^{n} \lambda_{i}\left(k_{i} \mid H(., x)\right)_{\mathcal{X}} \quad \lambda_{i} \in \mathbf{C} .
$$

$\mathrm{n}^{\circ}$ août 1975, R-2. 
$\sigma$ est appelée la fonction « spline » relative à $M_{n}(\alpha)$ et $\mathscr{H}$. $\operatorname{Si}\left(h \mid k_{i}\right)_{\mathcal{H}}=h\left(x_{i}\right)$, $x_{i} \in X, 1 \leqslant i \leqslant n$, on a :

$$
\begin{aligned}
\sigma & =\sum_{i=1}^{n} \lambda_{i} H\left(. ; x_{i}\right)=\sum_{i=1}^{n} \lambda_{i} H_{E} \varepsilon_{x_{i}}=H_{E}\left(\sum_{i=1}^{n} \lambda_{i} \varepsilon_{x_{i}}\right) \\
& =H\left(\sum_{i=1}^{n} \lambda_{i} \delta_{x_{i}}\right) .
\end{aligned}
$$

Application 3.1 : Méthode d'éléments finis (de Lagrange) d'ordre 1 et de degré 1 sur un maillage de type triangulaire.

(*) Soit $\Omega$ un ouvert de $\mathbf{R}^{n}$, borné et polyédrique, admettant une triangulation $\mathcal{C}$.

$\bar{\Omega}$ est alors la réunion d'un nombre fini de $n$-simplexes fermés (non dégénérés dans $\mathbf{R}^{n}$ ), d'intérieurs disjoints deux à deux, tels que chaque face d'un quelconque de ces $n$-simplexes soit ou bien une face commune à un autre $n$-simplexe de $\mathcal{G}$, ou bien une partie de la frontière de $\Omega$.

Désignons par $\mathfrak{C}^{0}$ le squelette d'ordre zéro de $\mathfrak{C}$, c'est-à-dire l'ensemble des sommets de $\mathcal{C}$ et par $|\mathfrak{G}|$ le polyèdre associé à $\mathscr{C}$; on a $: \bar{\Omega}=|\mathcal{G}|$.

Définition 3. 1: Soit $\zeta \in|\mathcal{G}|$. Notons $S(\zeta)$ le plus petit simplexe de $\mathcal{C}$ contenant $\zeta$.

On appellera coordonnées barycentriques généralisées de $\zeta$ relatives à $\mathcal{G}$, les scalaires $\zeta_{t}, t \in \mathcal{C}^{0}$, définis de la façon suivante :

(i) Si $t \notin S^{0}(\zeta)$ (c'est-à-dire si $t$ n'est pas un sommet de $S(\zeta)$ ), alors $\zeta_{t}=0$.

(ii) Si $t \in S^{\circ}(\zeta)$, alors $\zeta_{t}$ est la coordonnée barycentrique de $\zeta$ relative au sommet $t$ de $S(\zeta)$. On écrira $: \zeta=\left(\zeta_{t}\right)_{t \in \mathcal{C}^{\circ}}$.

Dans la suite, $\mathcal{H}$ sera l'espace de Hilbert défini dans l'exemple 1.2. On sait que si $e \in \mathcal{H}$, alors $e \in C^{0}[0,1]$ et $e(0)=0$.

$(* *)$ Soit :

$$
\pi=\left\{e \in C^{0}(\Omega) ; \forall \zeta \in|\mathcal{G}|, e(\zeta)=\sum_{t \in \mathscr{C}^{0}} e\left(\zeta_{t} ; t\right), e(. ; t) \in \mathcal{H}, t \in \mathcal{C}^{0}\right\} .
$$

Tout élément $e \in \nVdash$ est associé à une famille $(e(. ; t))_{t \in \mathcal{C}^{\circ}}$ d'éléments de $\mathcal{H}$. $\mathcal{K}$ a une structure d'espace vectoriel. Etant donné deux éléments quelconques $e$ et $f$ de $K$, associés respectivement aux familles $(e(. ; t))_{t \in \mathcal{G}^{0}}$ et $(f(. ; t))_{t \in \mathcal{C}^{0}}$ d'éléments de $\mathfrak{H}$, on vérifie facilement que l'application :

$$
\mid \begin{aligned}
& \mathcal{K} \times \mathcal{K} \mapsto \mathbf{R} \\
& (e, f) \mapsto((e \mid f))=\sum_{t \in \mathcal{C}^{\circ}} \int_{0}^{1} e^{\prime}(z ; t) \cdot f^{\prime}(z ; t) \mathrm{d} z=\sum_{t \in \mathcal{C}^{0}}(e(. ; t) \mid f(. ; t))_{\mathcal{H}}
\end{aligned}
$$

définit un produit scalaire sur $\pi$. 
Nous poserons : $\|||\|=\{((e \mid e))\} \frac{1}{2}$.

Pour la norme associée à ce produit scalaire, $\nVdash$ est un espace préhilbertien séparé. En effet :

$$
\|e\|=0 \Leftrightarrow\left(e^{\prime}(. ; t) \equiv 0, t \in \mathcal{C}^{0}\right) \Leftrightarrow\left(e(. ; t)=\theta_{\text {.T }}\right.
$$

$\left.(\operatorname{car} e(0 ; t)=0), t \in \mathcal{G}^{0}\right) \Leftrightarrow e=\theta_{\varkappa}$.

(***) Montrons que $\pi$ est complet. Soit $\left(e_{p}\right)_{p \in \mathbf{N}}$ une suite de Cauchy dans $(\mathcal{K},\|\mid\|)$. Chaque élément $e_{p}$ est associé à une famille $\left(e_{p}(\cdot ; t)\right)_{t \in \mathcal{G}^{\circ}},(p \in \mathbf{N})$ d'éléments de $\mathcal{H}$.

Chacune des suites $\left(e_{p}(. ; t)\right)_{p \in \mathbb{N}}, t \in \mathcal{C}^{0}$ est une suite de Cauchy dans $\mathscr{H}$ et converge donc vers un élément $\bar{e}(. ; t) \in \mathfrak{H}, t \in \mathcal{C}^{0}$.

L'élément $\bar{e} \in \mathcal{K}$ associé à la famille $(\bar{e}(. ; t))_{t \in \mathcal{C}^{\circ}}$ est la limite dans $(\mathcal{K},\|\| \|)$ de la suite $\left(e_{p}\right)_{p \in \mathbf{N}}$.

Soit $\zeta=\left(\zeta_{t}\right)_{t \in \mathcal{C}^{0}} \in|\mathcal{\zeta}|$.

Etant donné $e \in \Re$, on a :

$$
\begin{aligned}
|e(\zeta)| & =\left|\sum_{t \in \mathscr{C}^{\circ}} e\left(\zeta_{t} ; t\right)\right|=\left|\sum_{t \in \mathscr{C}^{0}} \int_{0}^{\zeta_{t}} e^{\prime}(z ; t) \mathrm{d} z\right| \\
& \leqslant \sum_{t \in \mathscr{C}^{\circ}} \int_{0}^{\zeta_{t}}\left|e^{\prime}(z ; t)\right| \mathrm{d} z \leqslant \sum_{t \in \mathscr{C}^{\circ}} \int_{0}^{1}\left|e^{\prime}(z ; t)\right| \mathrm{d} z \leqslant\left(\operatorname{Card}\left(\mathcal{C}^{0}\right)\right)^{\frac{1}{2}} \cdot \mid\|e\| \| .
\end{aligned}
$$

Donc

$$
\operatorname{Sup}_{\zeta \in \Omega}|e(\zeta)| \leqslant\left(\operatorname{Card}\left(\mathscr{C}^{0}\right)\right)^{\frac{1}{2}} \cdot\|e\|
$$

On en déduit que $\nVdash$ est un sous-espace hilbertien de $C^{0}(\bar{\Omega})$.

(****) Déterminons le noyau reproduisant de $\nVdash$.

On sait que c'est une fonction $\left(\zeta, \zeta^{\prime}\right) \mapsto K\left(\zeta, \zeta^{\prime}\right)$, symétrique, définie positive sur $\bar{\Omega} \times \bar{\Omega}$ telle que pour tout $\zeta \in \bar{\Omega}, K(., \zeta) \in \mathcal{K}$.

Soit $\left(K_{t}(., \zeta)\right)_{t \in \mathcal{C}^{\circ}}$ la famille d'éléments de $\mathfrak{H}$ à laquelle est associé $K(., \zeta)$.

Quels que soient $e \in K_{\text {et }} \zeta=\left(\zeta_{t}\right)_{t \in \mathcal{G}^{\circ}}$, on a :

$$
\begin{aligned}
e(\zeta) & =((e \mid K(., \zeta)))=\sum_{t \in \mathscr{C}^{0}}\left(e(. ; t) \mid K_{t}(. ; \zeta)\right)_{\mathcal{H}}=\sum_{t \in \mathscr{C}^{\circ}} e\left(\zeta_{t} ; t\right) \\
& =\sum_{t \in \mathscr{C}^{0}}\left(e(. ; t) \mid H\left(., \zeta_{t}\right)\right)_{\mathcal{H}^{\circ}} .
\end{aligned}
$$

On en déduit que pour tout $\zeta=\left(\zeta_{t}\right)_{t \in \mathcal{C}^{0}} \in \bar{\Omega}$, on a :

$$
K_{t}(., \zeta)=H\left(., \zeta_{t}\right), t \in \mathcal{C}^{0} \text {. }
$$

On montre facilement que la famille $(K(., \zeta))_{\zeta \in G^{\circ}}$ est libre. $\mathrm{n}^{\circ}$ août 1975, R-2. 
$(* * * *)$ Soit $M(\alpha)=\left\{e \in \mathcal{K} ; e(t)=\alpha(t),(\alpha(t) \in \mathbf{R}), t \in \mathscr{C}^{0}\right\} . M(\alpha)$ est une variété linéaire fermée dans $\pi$.

Soit $\sigma$ la fonction « spline » relative à $M(\alpha)$ et $\nVdash$ :

$$
\sigma=\sum_{\eta \in \mathcal{C}^{\circ}} \lambda_{\eta} K(., \eta), \quad \lambda_{n} \in \mathbf{R}, \quad \eta \in \mathcal{C}^{0}
$$

Ainsi quel que soit $\zeta=\left(\zeta_{t}\right)_{t \in \mathcal{C}^{\circ}} \in \bar{\Omega}$, on a :

$$
\begin{aligned}
\sigma(\zeta) & =\sum_{\eta \in \mathcal{C}^{\circ}} \lambda_{\eta}\left(\sum_{t \in \mathcal{C}^{\circ}} K_{t}\left(\zeta_{t}, \eta\right)\right)=\sum_{\eta \in \mathcal{C}^{\circ}} \lambda_{\eta}\left(\sum_{t \in \mathcal{C}^{\circ}} H\left(\zeta_{t}, \eta_{t}\right)\right) . \\
\text { Or } H\left(\zeta_{t}, \eta_{t}\right) & =\zeta_{t}-\left(\zeta_{t}-\eta_{t}\right)_{+}=\left\{\begin{array}{lll}
\zeta_{t} & \text { si } & \eta_{t}=1 \\
0 & \text { si } & \eta_{t}=0
\end{array}\right.
\end{aligned}
$$

On en déduit que $\sigma$ est une fonction affine sur chaque simplexe de $\mathscr{C}$.

Soit $\tau$ la fonction déterminée par la méthode des éléments finis d'ordre 1 qui prend les mêmes valeurs que $\sigma$ aux sommets de $\mathcal{~}$.

$\tau$ coïncide sur $\Omega$ avec la fonction « spline » $\sigma$.

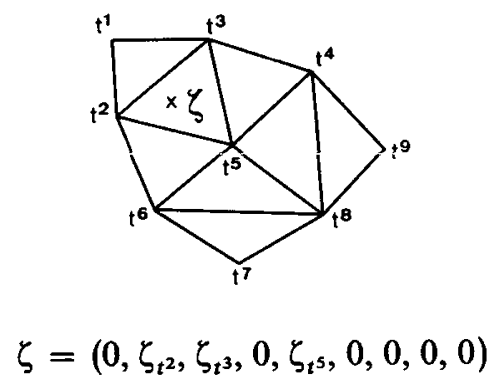

Notations 3.1 : Etant donné $n \in \mathbf{N}^{*}$, considérons :

(i) les ensembles $X_{i}=[0,1], 1 \leqslant i \leqslant n+1$

(ii) les espaces $E_{i}=C^{0}\left(X_{i}\right)$ et $\varepsilon_{i}=\mathbf{R}^{X_{i}}, 1 \leqslant i \leqslant n+1$.

Notons $X(n)$ (resp. $E(n) ; \varepsilon(n)$ ) le produit cartésien de $X_{1}, \ldots, X_{n+1}$ (resp. $E_{1}, \ldots, E_{n+1} ; \varepsilon_{1}, \ldots, \varepsilon_{n+1}$ ).

$E_{i}($ resp. $E(n))$ est un sous-espace admissible de $\varepsilon_{i}, 1 \leqslant i \leqslant n+1$, (resp. $\mathcal{E}(n))$.

Nous noterons $x=\left(x_{1}, \ldots, x_{n+1}\right)$ un point générique de $X(n)$ de composantes : $x_{i} \in X_{i}, 1 \leqslant i \leqslant n+1$.

$\mathcal{H}_{i}$ désignera un sous-espace hilbertien de $\mathcal{E}_{i}$, de noyau $H_{i}, 1 \leqslant i \leqslant n+1$. 
On notera $L_{n}$ la fonction suivante définie sur $(X(n))^{2}$ :

$$
(x, t) \mapsto L_{n}(x ; t)=\sum_{i=1}^{n+1} H_{i}\left(x_{i} ; t_{i}\right)
$$

$L_{n}$ est le noyau d'un sous-espace hilbertien $\mathcal{L}_{n}$ de $\mathcal{E}(n)$ (cf. exemple 2.3 ).

Etant donné $m \in \mathbf{N}^{*}$, on pose :

(*) $h=\frac{1}{m}$

(*) $J(n)=\left\{j=\left(j_{1}, \ldots, j_{n+1}\right) \in \mathbf{N}^{n+1} ; 0 \leqslant j_{i} \leqslant m\right\}$

(*) pour tout $j \in J(n), \hat{t}^{j}=\left(j_{1} h, \ldots, j_{n+1} h\right) ; \hat{t}^{j} \in X(n)$.

(*) $Y(n)=\left\{x=\left(x_{1}, \ldots, x_{n+1}\right) \in X(n) ; \sum_{i=1}^{n+1} x_{i}=1\right\}$

et $J_{1}(n)=\left\{j \in J ; \hat{t}^{j} \in Y(n)\right\}=\left\{j \in J ;|j|=\sum_{i=1}^{n+1} j_{i}=m\right\}$.

On supposera que $X(n)$ et $Y(n)$ sont munis de la topologie induite par celle de $\mathbf{R}^{n+1}$. $Y(n)$ est un $n$-simplexe.

$Y(n)$ a pour sommets les points $a_{i}=(0, \ldots, 0, \underset{i}{i}, 0, \ldots, 0) \in \mathbf{R}^{n+1}$.

Quel que soit $x=\left(x_{1}, \ldots, x_{n+1}\right) \in Y(n)$, on a :

$$
x=\sum_{i=1}^{n+1} x_{i} a_{i}
$$

On désignera par $\hat{\mathfrak{G}}(n)$ le complexe simplicial (géométrique) de sommets $\hat{t}^{j} \in Y(n),\left(j \in J_{1}(n)\right)$, ayant pour simplexes les sous-ensembles, d'intérieur non vide, de $Y(n)$, du type suivant :

$\left\{x=\left(x_{1}, \ldots, x_{n+1}\right) \in Y(n) ; x_{i}-j_{i} h>0\right.$ et $\left.x_{i}-\left(j_{i}+1\right) h<0,1 \leqslant i \leqslant n+1\right\}$.

$|\hat{\mathfrak{G}}(n)|$ désignant le polyèdre associé à $\hat{\mathfrak{G}}(n)$, on a :

$$
|\hat{\mathcal{G}}(n)|=Y(n)
$$

Soit $\hat{\Sigma}$ un $n$-simplexe (quelconque) de $\hat{\mathcal{C}}$ de sommets

$$
\hat{t}^{j(1)}, \ldots, \hat{t}^{j(n+1)} \quad \text { où } \quad \hat{t}^{j(i)}=\sum_{l=1}^{n+1} j_{l}(i) h \cdot a_{l}, \quad 1 \leqslant i \leqslant n+1 .
$$

$\mathrm{n}^{\circ}$ août 1975, R-2. 
Soit, d'autre part, $\hat{t}$ un point quelconque de $\Sigma$;

$$
\begin{gathered}
\hat{t}=\sum_{i=1}^{n+1} \lambda_{i}(\hat{\Sigma}) \cdot \hat{t}^{j(i)}=\sum_{i=1}^{n+1} t_{l} a_{l}, \\
\lambda_{i}(\hat{\Sigma}), a_{i} \in[0,1], \sum_{i=1}^{n+1} \lambda_{i}(\hat{\Sigma})=\sum_{i=1}^{n+1} a_{i}=1 .
\end{gathered}
$$

On a :

$$
t_{l}=\sum_{i=1}^{n+1} \lambda_{i}(\hat{\Sigma}) j_{l}(i) \cdot h, \quad 1 \leqslant l \leqslant n+1 .
$$

Posons : $J(\hat{\Sigma})=\left(j_{l}(i)\right)_{\substack{1 \leqslant i \leqslant n+1 \\ 1 \leqslant l \leqslant n+1}}$.

Comme les vecteurs $\hat{t}^{j(i)}, 1 \leqslant i \leqslant n+1$ sont linéairement indépendants, $J(\hat{\Sigma})$ est inversible :

$$
\lambda_{i}(\hat{\Sigma})=\sum_{i=1}^{n+1} \alpha_{i l} t_{l} \cdot \frac{1}{h} \quad \text { avec } \quad\left(\alpha_{i l}\right)_{\substack{1 \leqslant i \leqslant n+1 \\ 1 \leqslant i \leqslant n+1}}=(J(\Sigma))^{-1} .
$$

Application 3.2 : Méthode d'éléments finis (de Lagrange) d'ordre 1 sur un maillage de type rectangulaire.

Nous utiliserons ci-dessous, les notations 3.1. Nous supposerons que $H_{i}\left(x_{i} ; t_{i}\right)$ cst le noyau défini dans l'exemple 1.2. Soient $n, p \in \mathbf{N}^{*}$. La fonction $L$ définie sur $(X(m) \times X(p))^{2}$ par :

$$
((x, y),(t, v)) \rightarrow L(x, y ; t, v)=L_{n}(x ; t) \times L_{p}(y ; v)
$$

est le noyau d'un sous-espace hilbertien $\mathcal{L}$ de $\&(n) \varepsilon \mathcal{E}(p)$ (cf. exemples 2.3 et 2.4).

Notons $M$ la restriction de $L$ à $(Y(n) \times Y(p))^{2}$ et $\hat{\mathcal{G}}$ le produit cartésien des complexes simpliciaux $\hat{\mathscr{C}}(n)$ et $\hat{\mathscr{C}}(p)$, de sommets $\hat{t}^{i}, i \in J_{1}(n)$ et $\hat{v}^{j}, j \in J_{1}(p)$ respectivement.

Soient $x^{1}$ et $x^{2}$ deux éléments de $Y(n)$ tels que :

$$
\varepsilon_{i}\left(x_{i}-j_{i} h\right) \geqslant 0, \quad \varepsilon_{i}= \pm 1, \quad 1 \leqslant i \leqslant n+1, \quad l=1,2,
$$

$j$ étant un élément fixé de $J_{1}(n)$.

Quel que soit $\lambda \in[0,1]$, on a évidemment : $\lambda x^{1}+(1-\lambda) x^{2} \in Y(n)$. D'autre part, quels que soient $\lambda \in[0,1]$ et $i=1,2, \ldots,(n+1)$ :

$$
\begin{array}{r}
\left(\lambda x_{i}^{1}+(1-\lambda) x_{i}^{2}-j_{i} h\right)_{+}=\left(\lambda\left(x_{i}^{1}-j_{i} h\right)+(1-\lambda)\left(x_{i}^{2}-j_{i} h\right)\right)_{+} \\
=\lambda\left(x_{i}^{1}-j_{i} h\right)_{+}+(1-\lambda)\left(x_{i}^{2}-j_{i} h\right)_{+} .
\end{array}
$$


On en déduit facilement que pour tout

$$
\left(\hat{t}^{i}, \hat{v}^{j}\right) \in|\hat{\zeta}(n)| \times|\hat{\zeta}(p)| \quad\left(i \in J_{1}(n), j \in J_{1}(p)\right),
$$

l'application :

$$
\mid \begin{aligned}
& |\hat{\mathcal{C}}(n)| \times|\mathcal{G}(p)| \mapsto \mathbf{R} \\
& (x, y) \mapsto M\left(x, y ; \hat{t}^{i}, \hat{v}^{j}\right)
\end{aligned}
$$

se réduit à une fonction affine par rapport à $x$ (resp. $y$ ) sur la fermeture de tout ensemble de la forme : $\hat{\Sigma}_{1} \times\{v\}\left(\right.$ resp. $\left.\{t\} \times \hat{\Sigma}_{2}\right)$ où $\hat{\Sigma}_{1}$ (resp. $\hat{\Sigma}_{2}$ ) est un $n$-simplexe (resp. $p$-simplexe) de $\hat{\mathfrak{G}}(n)$ (resp. $\hat{\mathfrak{C}}(p)$ ) et $v \in|\hat{\mathcal{G}}(p)|$ (resp. $t \in|\hat{\mathfrak{G}}(n)|$ ).

Soit $\psi$ une application inversible de $|\hat{G}(n)| \times|\hat{G}(p)|$ dans $\mathbf{R}^{n+p}$, simpliciale par rapport à chacun de ses arguments (cf. fig. 3.2).

$\mathscr{G}=\psi(\hat{G})$ est un complexe simplicial (géométrique) de $\mathbf{R}^{n+p}$ (de même dimension que $\hat{\mathfrak{G}}$ ).

$\mathcal{C}$ a pour sommets les points $\eta^{i j}=\psi\left(\hat{t}^{i}, \hat{v}^{j}\right), i \in J_{1}(n), j \in J_{1}(p)$ et pour $n$-simplexes les ensembles $\psi\left(\hat{\Sigma}_{1} \times\left\{\hat{v}^{j}\right\}\right)$ et $\psi\left(\left\{\hat{t}^{i}\right\} \times \hat{\Sigma}_{2}\right)$.

Posons : $Z=\psi(|\hat{\mathcal{G}}(n)| \times|\hat{\mathfrak{G}}(p)|)$.

Désignons par $K$ la fonction numérique définie sur $Z^{2}$ par :

$$
\forall \zeta, \eta \in Z, K(\zeta ; \eta)=M\left(\psi^{-1}(\zeta) ; \psi^{-1}(\eta)\right)
$$

$K$ définit un sous-espace hilbertien $\mathscr{H}$ de $\mathbf{R}^{z}$.

On vérifie facilement que pour tout

$$
\eta^{i j} \in Z, i \in J_{1}(n), j \in J_{1}(p),
$$

l'application : $\mid \begin{aligned} & Z \mapsto \mathbf{R} \\ & \zeta \mapsto K\left(\zeta ; \eta^{i j}\right)\end{aligned}$ se réduit à une fonction affine sur la fermeture de tout ensemble de la forme :

$$
\psi\left(\hat{\Sigma}_{1} \times\{v\}\right)\left(\text { resp. } \psi\left(\{t\} \times \hat{\Sigma}_{2}\right)\right) .
$$

Il en est de même de la fonction "spline » :

$$
\sigma=\sum_{i \in J_{1}(n), j \in J_{1}(p)} \lambda_{i j} K\left(. ; \eta^{i j}\right), \lambda_{i j} \in \mathbf{R} .
$$

De plus, $\sigma \in C^{0}(Z)$.

On montre facilement que $\sigma$ est déterminée de manière unique par ses valeurs aux sommets de $\mathfrak{C}$.

Soit $\Omega$ la réunion d'une famille finie d'ensembles du type $: \psi\left(\left|\hat{\Sigma}_{1}\right| \times\left|\hat{\Sigma}_{2}\right|\right)$, et $\bar{\Omega}$ la fermeture de $\Omega$.

$n^{\circ}$ août 1975, R-2. 
Soit $\tau$ la fonction déterminée par la méthode des éléments finis d'ordre 1 qui prend les mêmes valeurs que $\sigma$ aux sommets de $\mathcal{C}$ contenus dans $\bar{\Omega}$.

$\tau$ coïncide avec $\sigma$ sur $\bar{\Omega}$, comme on peut le vérifier facilement.
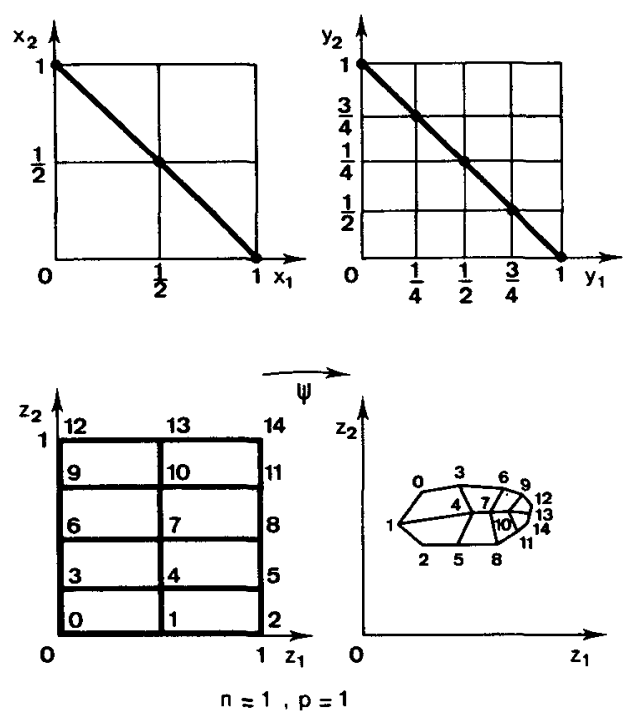

Figure 3.2

4. Puissances tensorielles symétriques d'espaces hilbertiens et méthodes d'éléments finis

Lemme 4.1 : Soit $E$ un espace vectoriel sur $C$ et $E^{*}$ son dual algébrique.

(i) Soient $e_{1}, \ldots, e_{n}, n$ éléments linéairement indépendants de $E$. Etant donné $k \in \mathbf{N}^{*}$, posons : $k_{*}=C_{n+k-1}^{k}=C_{k+n-1}^{n-1} ; k_{*}$ est le nombre de monômes d'un polynôme homogène de degré $k$ à $n$ variables.

Soit $\left\{\alpha^{j}=\left(\alpha_{1}^{j}, \ldots, \alpha_{n}^{j}\right) ; 1 \leqslant j \leqslant k_{*}\right\}$ une famille d'éléments de $\mathbf{C}^{n}$.

Pour que la famille $\left\{\left(\sum_{i=1}^{n} \alpha_{i}^{j} e_{i}\right)^{\vee k} ; 1 \leqslant j \leqslant k_{*}\right\}$ soit libre dans $E^{\vee k}$, il faut et il suffit que les points : $\alpha^{1}, \alpha^{2}, \ldots, \alpha^{k}$ forment un système unisolvent dans $\mathbf{C}^{n}$ pour les polynômes homogènes de degré $k$.

(ii) Soient $e_{1}^{\prime}, \ldots, e_{n}^{\prime} \in E^{\prime}$ tels que :

$$
\Delta_{n}=\operatorname{det}\left(\left[\left\langle e_{i}, e_{j}^{\prime}\right\rangle\right]_{1 \leqslant j \leqslant n}\right) \neq 0 .
$$


Il existe un seul système de $k_{*}$ polynômes homogènes

$$
\Pi_{j}^{k}\left(\begin{array}{l}
e_{1}, \ldots, e_{n} \\
e_{1}^{\prime}, \ldots, e_{n}^{\prime}
\end{array}\right)=\sum_{|\beta|=k} \mu_{\beta}^{j}\left(\left(e_{1}^{\prime}\right)^{\vee \beta_{1}} \vee \ldots \vee\left(e_{n}^{\prime}\right)^{\vee \beta_{n}}\right), \mu_{\beta}^{j} \in \mathbf{C},|\beta|=\sum_{i=1}^{n} \beta_{i}
$$

tels que : $\left\langle\Pi_{j}^{k}\left(\begin{array}{l}e_{1}, \ldots, e_{n} \\ e_{1}^{\prime}, \ldots, e_{n}^{\prime}\end{array}\right),\left(\sum_{i=1}^{n} \alpha_{i}^{l} e_{i}\right)^{v k}\right\rangle=\delta_{j l}, \quad 1 \leqslant j, l \leqslant k_{*}$.

\section{Preuve :}

(i) Notons $\theta_{k}$ l'élément neutre de $E^{\vee k}$.

Pour que les éléments $\left(\sum_{i=1}^{n} \alpha_{i}^{j} \cdot e_{i}\right)^{\vee k}, 1 \leqslant j \leqslant k_{*}$ soient linéairement indépendants dans $E^{\vee k}$, il faut et il suffit que :

$$
\begin{gathered}
\sum_{1 \leqslant j \leqslant k_{*}} \lambda_{j}\left(\sum_{i=1}^{n} \alpha_{i}^{j} e_{i}\right)^{\vee k}=\theta_{k} \Rightarrow \lambda_{j}=0, \quad 1 \leqslant j \leqslant k_{*} . \\
\text { Or, } \sum_{1 \leqslant j \leqslant k_{*}} \lambda_{j}\left(\sum_{i=1}^{n} \alpha_{i}^{j} e_{i}\right)^{\vee k} \\
=\sum_{1 \leqslant j \leqslant k_{*}} \lambda_{j} \cdot\left[\sum_{|\beta|=k} c_{\beta}\left(\alpha_{1}^{j}\right)^{\beta_{1}} \ldots\left(\alpha_{n}^{j}\right)^{\beta_{n}}\left(e_{1}^{\vee \beta_{1}} \vee \ldots \vee e_{n}^{\vee \beta_{n}}\right)\right] \\
=\sum_{|\beta|=k} \sum_{1<j<k_{*}} \lambda_{j}\left(\alpha_{1}^{j}\right)^{\beta_{1}} \ldots\left(\alpha_{n}^{j}\right)^{\beta_{n}} c_{\beta}\left(e_{1}^{\beta_{1}} \vee \ldots \vee e_{n}^{\beta_{n}}\right) .
\end{gathered}
$$

Comme $c_{\beta} \neq 0,|\beta|=k$, on a :

$$
\sum_{1 \leqslant j \leqslant k_{*}} \lambda_{j}\left(\sum_{i=1}^{n} \alpha_{i}^{j} e_{i}\right)^{k}=\theta_{k} \Leftrightarrow \sum_{1 \leqslant j \leqslant k_{*}} \lambda_{j}\left(\alpha_{1}^{j}\right)^{\beta_{1}} \ldots\left(\alpha_{n}^{j}\right)^{\beta_{n}}=0,|\beta|=k .
$$

Soit $D(k)$ le déterminant de la matrice ayant pour lignes (resp. colonnes) les éléments de la forme :

$$
\left.\left(\alpha_{1}^{j}\right)^{\beta_{1}} \ldots\left(\alpha_{n}^{j}\right)^{\beta_{n}},|\beta|=k \text { (resp. } 1 \leqslant j \leqslant k_{*}\right) .
$$

Pour que $\lambda_{j}=0,1 \leqslant j \leqslant k_{*}$, il faut et il suffit que $D(k) \neq 0$, c'est-à-dire que les points $\alpha^{1}, \alpha^{2}, \ldots, \alpha^{k *}$ forment un système unisolvent dans $\mathbf{C}^{n}$ pour les polynômes homogènes de degré $k$.

(ii)

$$
\begin{aligned}
\left\langle\Pi_{j}^{k},\left(\sum_{i=1}^{n} \alpha_{i}^{l} e_{i}\right)^{\vee k}\right\rangle & =\left\langle\sum_{|\beta|=k} \mu_{\beta}^{j}\left(\left(e_{1}^{\prime}\right)^{\vee \beta_{1}} \vee \ldots \vee\left(e_{n}^{\prime}\right)^{\vee \beta_{n}}\right),\left(\sum_{i=1}^{n} \alpha_{i}^{l} e_{i}\right)^{\vee k}\right\rangle \\
& =\sum_{|\beta|=k} \mu_{\beta}^{j}\left(k ! \prod_{1 \leqslant s \leqslant n}\left(-\sum_{1 \leqslant i \leqslant n} \alpha_{i}^{l}\left\langle e_{i}, e_{s}^{\prime}\right\rangle\right)^{\beta_{i}}\right), \quad 1 \leqslant j, l \leqslant k^{*} .
\end{aligned}
$$

$n^{\circ}$ août 1975, R-2. 
Posons :

$$
\begin{gathered}
\gamma_{s}^{j}=\sum_{1 \leqslant i \leqslant n} \alpha_{i}^{j}\left\langle e_{i}, e_{s}^{\prime}\right\rangle, \\
\alpha_{j}=\left(\begin{array}{c}
\alpha_{1}^{j} \\
\vdots \\
\alpha_{n}^{j}
\end{array}\right), \quad \gamma^{j}=\left(\begin{array}{c}
\gamma_{1}^{j} \\
\vdots \\
\gamma_{n}^{j}
\end{array}\right), \quad A=\left(\begin{array}{cc}
\left\langle e_{1}, e_{1}^{\prime}\right\rangle \ldots\left\langle e_{n}, e_{1}^{\prime}\right\rangle \\
\vdots & \vdots \\
\left\langle e_{1}, e_{n}^{\prime}\right\rangle \ldots\left\langle e_{n}, e_{n}^{\prime}\right\rangle
\end{array}\right)
\end{gathered}
$$

On a $: \gamma^{j}=A \alpha^{j}$ avec $\operatorname{det}(A)=\Delta_{n} \neq 0$.

On sait qu'il existe un seul système de $k_{*}$ polynômes homogènes de $n$ variables complexes $P_{j}, 1 \leqslant j \leqslant k_{*}$ tels que :

$$
P_{j}\left(\alpha^{l}\right)=\delta_{j l}, ; 1 \leqslant j, l \leqslant k_{*} .
$$

La transformation linéaire : $\begin{aligned} \mathbf{C}^{n} & \rightarrow \mathbf{C}^{n} \\ z & \rightarrow A z=v\end{aligned}$, étant inversible applique l'espace vectoriel des polynômes homogènes à $n$ variables complexes, de degré $k$, sur lui-même.

On en déduit que les polynômes homogènes $Q_{l}, 1 \leqslant l \leqslant k_{*}$ définis par : $Q_{l}(z)=P_{l}\left(A^{-1} z\right)$ sont linéairement indépendants.

$$
\text { Or } Q_{l}\left(\gamma^{j}\right)=P_{l}\left(A^{-1} A \alpha^{j}\right)=P_{l}\left(\alpha^{j}\right)=\delta_{l j} \quad, \quad 1 \leqslant l, j \leqslant k_{*} .
$$

Il en résulte que les points $\gamma^{1}, \ldots, \gamma^{k}$ forment un système unisolvent pour les polynômes homogènes de $n$ variables complexes, de degré $k$.

Lorsque $j\left(1 \leqslant j \leqslant k_{*}\right)$ est fixé, il existe donc un seul système de scalaires $\mu_{\beta}^{j},|\boldsymbol{\beta}|=k$, tels que :

$$
(k !)\left(\sum_{|\beta|=k} \mu_{\beta}^{j}\left(\gamma_{1}^{l}\right)^{\beta_{1}} \ldots\left(\gamma_{n}^{l}\right)^{\beta_{n}}\right)=\delta_{j l}, \quad 1 \leqslant j, l \leqslant k_{*} \cdot \|
$$

\section{EXEMPLE 4.1:}

Lemme 4.1 * L'ensemble : $\left\{\alpha=\left(\alpha_{1}, \ldots, \alpha_{n}\right) \in \mathbf{N}^{n} ;|\alpha|=k\right\}$ est un système de points unisolvent dans $\mathbf{C}^{n}$ pour les polynômes homogènes de degré $k$.

** Avec les mêmes hypothèses et notations que dans le lemme 4.1 (i), posons :

$$
\begin{array}{r}
u_{j}=\frac{1}{\Delta_{n}}\left[\sum_{i=1}^{n}(-1)^{i+j}\left\langle e_{1} \wedge \ldots \wedge e_{i} \wedge \ldots \wedge e_{n}, e_{1}^{\prime} \wedge \ldots \wedge e_{j}^{\prime} \wedge \ldots \wedge e^{n}\right\rangle e_{i}\right], \\
1 \leqslant j \leqslant n, \\
u_{0}=\sum_{j=1}^{n} u_{j} \text { On a }:\left\langle u_{j}, e_{k}^{\prime}\right\rangle=\delta_{j k}\left\langle u_{0}, e_{k}^{\prime}\right\rangle=1, \quad 1 \leqslant j, k \leqslant n .
\end{array}
$$


Etant donné $\beta=\left(\beta_{1}, \ldots, \beta_{n}\right) \in \mathbf{N}^{n}$ tel que $|\beta|=k$, soit $\Pi_{\beta}^{k}\left(\begin{array}{l}e_{1}, \ldots, e_{n} \\ e_{1}^{\prime}, \ldots, e_{n}^{\prime}\end{array}\right)=\frac{1}{\beta !} \bigvee_{i=1}^{n} \bigvee_{j=0}^{\beta_{i}-1}\left(k u_{i}-j u_{0}\right)$, où $\beta !=\beta_{1} ! \ldots \beta_{n} !$

On a : $\left\langle\Pi_{\beta}^{k},\left(\sum_{j=1}^{n} \frac{\alpha_{j} e_{j}^{\prime}}{k}\right)^{v k}\right\rangle=\delta_{\alpha \beta}$ où $\alpha=\left(\alpha_{1}, \ldots, \alpha_{n}\right) \in \mathbf{N}^{n}$ et $|\alpha|=k$. Il en résulte que les polynômes $\Pi_{\beta}^{k}$ sont les seuls polynômes homogènes de degré $k$ vérifiant les conditions ci-dessus.

Preuve : ${ }^{*}$ Considérons $n$ variables réelles $x_{1}, \ldots, x_{n}$ indépendantes. (On vérifie facilement que) les $n$ applications de $\mathbf{R}$ dans $\mathbf{R}_{+}$:

$$
x_{1} \rightarrow e^{x_{1}}, \ldots, x_{n} \rightarrow e^{x_{n}}
$$

sont linéairement indépendantes.

Il en est de même des applications de $\mathbf{R}^{n}$ dans $\mathbf{R}_{+}$:

$$
\begin{gathered}
\quad\left(x_{1}, \ldots, x_{n}\right) \rightarrow\left(e^{x_{1}}\right)^{\alpha_{1}} \ldots\left(e^{x_{n}}\right)^{\alpha_{n}}=e^{\sum_{i=1}^{n} \alpha_{i} x_{i}},|\alpha|=k . \\
\text { Soit } V=\sum_{|\alpha|=k} \mu_{\alpha} e^{\sum_{i=1}^{n} \alpha_{i} x_{i}}, \mu_{\alpha} \in \mathbf{C} . \\
\frac{\partial^{k} v}{\partial x_{1}^{\beta_{1}} \ldots \partial x_{n}^{\beta_{n}}}=\sum_{|\alpha|=k} \mu_{\alpha}\left(\alpha_{1}\right)^{\beta_{1}} \ldots\left(\alpha_{n}\right)^{\beta_{n}} \cdot e^{\sum_{i=1}^{n} \alpha_{i} x_{i}},|\beta|=k .
\end{gathered}
$$

On établit sans difficulté que : $\left(\left\{\alpha=\left(\alpha_{1}, \ldots, \alpha_{n}\right) \in \mathbf{C}^{n} ;|\alpha|=k\right\}\right.$ est un système de points unisolvent pour les polynômes homogènes à $n$ variables complexes de degré $k$ )

$$
\begin{aligned}
& \Leftrightarrow\left(\sum_{|\beta|=k} \mu_{\beta} \cdot \alpha_{1}^{\beta_{1}} \ldots \alpha_{n}^{\beta_{n}}=0,|\alpha|=k \Rightarrow \mu_{\beta}=0,|\beta|=k\right) \\
& \Leftrightarrow\left(\sum_{|\alpha|=k} \mu_{\alpha} \cdot \alpha_{1}^{\beta_{1}} \ldots \alpha_{n}^{\alpha_{n}}=0,|\beta|=k \Rightarrow \mu_{\alpha}=0,|\alpha|=k\right) \\
& \Leftrightarrow\left(\sum_{|\alpha|=k} \mu_{\alpha} \cdot \alpha_{1}^{\beta_{1}} \ldots \alpha_{n}^{\beta_{n}} \cdot e^{\sum_{i=1}^{n} \alpha_{i}} \equiv 0,|\beta|=k \Rightarrow \mu_{\alpha}=0,|\alpha|=k\right) \\
& \Leftrightarrow\left(\frac{\partial^{k} v}{\partial x_{1}^{\beta_{1}} \ldots \partial x_{n}^{\beta_{n}}} \equiv 0,|\beta|=k \Rightarrow \mu_{\alpha}=0,|\alpha|=k\right) .
\end{aligned}
$$

$\mathrm{n}^{\circ}$ août $1975, \mathrm{R}-2$. 
Or si $\frac{\partial^{k} v}{\partial x_{1}^{\beta_{1}} \ldots \partial x_{n}^{\beta_{n}}} \equiv 0,|\beta|=k, v$ est un polynôme de degré inférieur ou égal à $k$. On en déduit facilement que $v \equiv 0$, c'est-à-dire que $\mu_{\alpha}=0,|\alpha|=k$.

$$
\begin{aligned}
* *\left\langle\Pi_{\beta}^{k},\left(\sum_{i=1}^{n} \frac{\alpha_{i} e_{i}^{\prime}}{k}\right)^{v k}\right\rangle & =\frac{1}{\beta !} \prod_{s=1}^{n} \sum_{j=0}^{\beta_{s}+1}\left\langle k u_{s}-j u_{0}, \sum_{i=1}^{n} \frac{\alpha_{i} e_{i}^{\prime}}{k}\right\rangle \\
& =\frac{1}{\beta !} \prod_{s=1}^{n} \prod_{j=0}^{\beta_{s}+1}\left(\frac{k \alpha_{s}}{k}-j \frac{\sum_{i=1}^{n} \alpha_{i}}{k}\right) \\
& =\frac{1}{\beta !} \prod_{s=1}^{n} \prod_{j=0}^{\beta_{s}+1}\left(\alpha_{s}-j\right) \operatorname{car} \sum_{i=1}^{n} \alpha_{i}=k .
\end{aligned}
$$

S'il existe $i_{0}, 1 \leqslant i_{0} \leqslant n$ tel que : $\alpha_{i_{0}} \neq \beta_{i_{0}}$, alors il existe $i_{1}, 1 \leqslant i_{1} \leqslant n$ tel que $0 \leqslant \alpha_{i_{1}} \leqslant \beta_{i_{1}}-1$ et

$$
\left\langle\Pi_{\beta}^{k},\left(\sum_{j=1}^{n} \frac{\alpha_{j} e_{j}^{\prime}}{k}\right)^{v k}\right\rangle=0 .
$$

Si $\alpha_{i}=\beta_{i}, 1 \leqslant i \leqslant n$, on a :

$$
\left\langle\Pi_{\beta}^{k},\left(\sum_{j=1}^{n} \frac{\alpha_{j} e_{j}^{\prime}}{k}\right)^{\vee k}\right\rangle=\frac{1}{\beta !}\left(\prod_{s=1}^{n} \beta_{s}\left(\beta_{s}-1\right) \ldots 2.1\right)=1 . \|
$$

Définition 4.1 : Soit $E$ un espace vectoriel sur C. Etant donné un simplexe géométrique $\Sigma$ de sommets $e_{1}, \ldots, e_{q} \in E$ et de dimension $(q-1),\left(\tilde{q} \in \mathbf{N}^{*}\right)$, nous appellerons treillis barycentrique d'ordre $k \in \mathbf{N}^{*}$, associé $a ̀ \Sigma$ et nous le noterons $\Sigma_{k}$, l'ensemble :

$$
\Sigma_{k}=\left\{\sum_{i=1}^{q} \frac{\alpha_{i}}{k} e_{i} \in E ; \alpha=\left(\alpha_{1}, \ldots, \alpha_{q}\right) \in \mathbf{N}^{q},|\alpha|=k\right\} .
$$

Soit $\mathfrak{C}$ un complexe simplicial géométrique de sommets $e_{1}, \ldots, e_{n} \in E$. On appellera treillis barycentrique d'ordre $k$ associé à $\mathfrak{C}$ et on le notera $\mathfrak{C}_{k}$, l'ensemble :

$$
\widetilde{C}_{k}=\bigcup_{\Sigma \in \mathcal{C}} \Sigma_{k} \text {, où } \Sigma \text { désigne un simplexe de } \mathscr{C} \text {. }
$$

Tout élément $t \in \mathscr{C}_{k}$ s'écrit sous la forme :

$$
t=\sum_{i=1}^{n} \frac{\alpha_{i}}{k} e_{i} \text { où } \alpha=\left(\alpha_{1}, \ldots, \alpha_{n}\right) \in \mathbf{N}^{n} \text { et }|\alpha|=k .
$$

Théorème 4.1 : Soient $E$ un espace vectoriel sur $\mathrm{C}$ et $e_{1}, \ldots, e_{n}, n$ éléments linéairement indépendants de $E$. 
Soit, d'autre part, $\mathfrak{C}$ un complexe simplicial de sommets $e_{1}, \ldots, e_{n} \in E$. La famille $\left\{t^{\vee k} \in E^{\vee k} ; t \in T_{k}\right\}$ forme un système libre dans $E^{\vee k}$.

Preuve : Résulte immédiatement du lemme 4.2.

Application 4.1: Méthode d'éléments finis (de Lagrange) d'ordre $k \in \mathbf{N}$, $k \geqslant 2$, sur un maillage de type triangulaire.

a) Éléments finis rectilignes

Nous ferons ci-dessous les mêmes hypothèses et nous utiliserons les mêmes notations que dans l'application 3.1. Notons $K^{\vee k}$ le noyau du sousespace hilbertien $\mathcal{H}^{\vee k}$ de $\left(C^{0}(\bar{\Omega})\right)^{\vee k}$. Désignons par $\delta_{\zeta} \in\left(C^{0}(\bar{\Omega})\right)^{\prime}$ la mesure de Dirac au point (quelconque) $\zeta \in \bar{\Omega}$.

On sait que $: \forall \zeta, \eta \in \bar{\Omega}, K(\zeta, \eta)=\left\langle K \delta_{\eta}, \delta_{\zeta}\right\rangle$ et que $: \forall \zeta^{1}, \ldots, \zeta^{k}, \eta^{1}, \ldots, \eta^{k} \in \bar{\Omega}$,

$$
\begin{aligned}
\left\langle K^{\vee k}\left(\delta_{\eta^{1}} \vee \ldots \vee \delta_{\eta^{k}}\right), \delta_{\zeta^{1}} \vee \ldots \vee \delta_{\zeta^{k}}\right. & \\
& =\frac{1}{k !} \sum_{\rho \in \mathcal{G}_{k}}\left\langle K \delta_{\eta^{1}}, \delta_{\zeta^{\rho(1)}}\right\rangle \ldots\left\langle K \delta_{\eta^{k}}, \delta_{\zeta^{\rho(k)}}\right\rangle \\
& =\frac{1}{k !} \sum_{\rho \in \mathcal{G}_{k}} K\left(\eta^{1}, \zeta^{\rho(1)}\right), \ldots, K\left(\eta^{k}, \zeta^{\rho(k)}\right) \\
& =\left\langle K \delta_{\eta^{1}} \ldots K \delta_{\eta^{k}}, \delta_{\zeta^{\rho(1)}} \ldots \delta_{\zeta^{\rho(k)}}\right\rangle .
\end{aligned}
$$

Soit $\mathfrak{G}_{\boldsymbol{k}}$ le treillis barycentrique d'ordre $k$ associé à $\mathfrak{C}$. Quel que soit $\zeta \in \mathscr{C}_{k}$ (notons $S_{k}(\zeta)$ le treillis barycentrique d'ordre $k$ associé à $S(\zeta)$ ) et posons :

$$
\begin{aligned}
& h(\zeta)=K^{\vee k}\left(\sum_{t \in S_{k}(\zeta)} \frac{\alpha_{t}(\zeta)}{k} \delta_{t}\right)^{\vee k} \in \mathcal{K}^{\vee k}, \alpha_{t}(\zeta) \in \mathbf{N}, t \in S_{k}(\zeta), \sum_{t \in S_{k}(\zeta)} \alpha_{t}(\zeta)=k . \\
& \text { Comme } h(\zeta)=\left(K\left(\sum_{t \in S_{k}(\zeta)} \frac{\alpha_{t}(\zeta)}{k} \delta_{t}\right)\right)^{\vee k}=\left(\sum_{t \in S_{k}(\zeta)} \frac{\alpha_{t}(\zeta)}{k} K \delta_{t}\right)^{\vee k} \text {, on déduit du }
\end{aligned}
$$

théorème 4.1 que la famille $(h(\zeta))_{\zeta \in \zeta k}$ est libre dans $\mathcal{H}^{v k}$.

Etant donné deux éléments $\zeta$ et $\eta \in \mathscr{C}_{k}$, on a

$$
\begin{aligned}
((h(\zeta) \mid h(\eta)))_{K^{v k}}=\left\langle h(\zeta),\left(\sum_{t \in S_{k}(\eta)} \frac{\alpha_{t}(\eta)}{k} \delta_{t}\right)^{v k}\right\rangle & \\
= & \left(\left\langle K\left(\sum_{t \in S_{k}(\zeta)} \frac{\alpha_{t}(\zeta)}{k} \delta_{t}\right), \sum_{t \in S_{k}(\eta)} \frac{\alpha_{t}(\eta)}{k} \delta_{t}\right\rangle\right)^{k} \\
& =\left(\left\langle K\left(\sum_{t \in S_{k}(\zeta)} \frac{\alpha_{t}(\zeta)}{k} \delta_{t}\right), \sum_{t \in S_{k_{k}(\eta)}} \frac{\alpha_{t}(\eta)}{k} \delta_{t}\right\rangle\right)^{k}
\end{aligned}
$$

$\mathrm{n}^{\circ}$ août 2975 , R-2. 
car l'application

$$
\mid \begin{aligned}
& \mathbf{R}^{|\mathfrak{C}|} \mapsto \mathbf{R} \\
& \zeta \mapsto K(\zeta, t)
\end{aligned}, \quad t \in \mathcal{C}^{0}
$$

se réduit à une fonction affine sur la fermeture de chaque $n$-simplexe de $\mathfrak{C}$. Ainsi :

$$
(h(\zeta) \mid h(\eta))_{\pi^{\vee k}}=\left\langle h(\zeta),\left(\delta_{\eta}\right)^{\vee k}\right\rangle .
$$

Soit $\sigma$ la restriction à la diagonale de $\left(|\mathcal{G}|^{k}\right)$ de la fonction "spline » $\chi=\sum_{\zeta \in \mathscr{C}_{k}} \mu(\zeta) \cdot h(\zeta), \mu(\zeta) \in \mathbf{R}$, relative à $\varkappa^{\vee k}$ (et aux points $h(\zeta), \zeta \in \mathscr{C}_{k}$ ).

Quel que soit $\eta \in \bar{\Omega}$, on a :

$$
\sigma(\eta)=\left\langle\chi,\left(\delta_{\eta}\right)^{v k}\right\rangle=\sum_{\zeta \in \mathcal{G}^{k}} \mu(\zeta)\left[\sum_{t \in S_{k}(\zeta)} \frac{\alpha_{t}(\zeta)}{k} K(t, \eta)\right]^{k} .
$$

$\sigma$ se réduit donc à un polynôme de degré (inférieur ou) égal à $k$ sur chaque $n$-simplexe de $\mathscr{C}$. De plus, $\sigma \in C^{0}(|\mathfrak{G}|)$.

D'autre part, si $\eta \in \mathcal{C}_{k}$, on a :

$$
\sigma(\eta)=\sum_{\zeta \in \mathscr{G}_{k}} \mu(\zeta)\left\langle h(\zeta),\left(\zeta_{\eta}\right)^{\vee k}\right\rangle=\sum_{\zeta \in \mathscr{G}_{k}} \mu(\zeta)((h(\zeta) \mid h(\eta)))_{K^{\vee k}}
$$

Il en résulte que $\sigma$ est déterminé de manière unique par la donnée de ses valeurs aux points de $\mathfrak{G}_{\boldsymbol{k}}$.

Si $\tau$ est la fonction d'éléments finis d'ordre $k$ qui prend les mêmes valeurs que $\sigma$ aux points de $\mathcal{C}_{k}$ contenus dans $\bar{\Omega}, \tau$ coïncide avec $\sigma$ sur $\bar{\Omega}$.

b) Eléments finis courbes

Soit : $\zeta \mapsto \sigma(\zeta), \eta \in \mathscr{C}_{k}$ la restriction à la diagonale de $|\mathcal{\zeta}|^{k}$ de la fonction « spline " : $\chi=\sum_{t \in \mathcal{C}_{\mathrm{k}}} \mu(t) \cdot h(t), \mu(t) \in \mathbf{R}$, définie en a) telle que :

$$
\sigma_{\eta}\left(\eta^{\prime}\right)=\left\{\begin{array}{lll}
0 & \text { si } & \eta \neq \eta^{\prime} \\
1 & \text { si } & \eta=\eta^{\prime}
\end{array}, \eta, \eta^{\prime} \in \mathcal{C}_{k} .\right.
$$

Soit $\tilde{\mathscr{C}}_{k}=(v(\eta))_{\eta \in \mathcal{C}_{k}}$ un système de points de $\mathbf{R}^{n}$ tel que l'application $\tilde{\psi}_{k}$ de $\mathscr{C}_{k}$ dans $\mathbf{R}^{n}$ définie par : $\widetilde{\psi}_{k}=\sum_{\eta \in \mathscr{C}_{k}} \sigma_{\eta} \cdot v(\eta)$ soit inversible.

L'image de $\mathscr{C}$ par $\tilde{\psi}_{k}$ est composée d'éléments finis courbes.

Posons : $|\tilde{\mathfrak{G}}|=\tilde{\Psi}_{k}(|\tilde{\mathfrak{G}}|)$ et, $\forall z \in|\tilde{\mathfrak{G}}|, \tilde{h}(z)=h\left(\tilde{\Psi}_{k}^{-1}(z)\right)$. Alors la restriction $\tilde{\sigma}$ à la diagonale de $|\tilde{G}|^{k}$ de la fonction « spline » :

$$
\tilde{\chi}=\sum_{z \in k} \tilde{\mu}(z) \cdot \tilde{h}(z), \tilde{\mu}(z) \in \mathbf{R},
$$

est une fonction d'interpolation de Lagrange d'ordre $k$ associée à la famille d'éléments finis courbes qui est l'image de $\mathscr{C}$ par $\Psi_{k}$.

Revue Française d'Automatique, Informatique et Recherche Opérationnelle 
Notons qụe $\tilde{\sigma}$ est définie de manière unique par la donnée de ses valeurs aux points de $\tilde{\mathscr{C}}_{k}$.

Application 4.2 : Méthode d'éléments finis d'Hermite sur un maillage de type triangulaire

Considérons un $n$-simplexe $\Sigma$ (quelconque) de $\mathscr{C}$ de sommets $t^{1}, \ldots, t^{n+1} \in \mathcal{C}^{0}$. Notons $P_{\Sigma}$ le polynôme de degré minimal sur $\mathbf{R}^{n}$ tel que : $\forall t \in \Sigma_{k}, P_{\Sigma}(t)=\sigma(t)$.

$P_{\Sigma}$ coïncide avec $\sigma$ sur $|\bar{\Sigma}|$.

a) Dérivées d'ordre 1. Éléments finis de classe $C^{0}$

Supposons maintenant que $P_{\Sigma}$ soit déterminé de manière unique par la donnée simultanée de ses valeurs $P_{\Sigma}\left(t^{j}\right)$ aux sommets de $\Sigma$ et de ses dérivées premières $P_{\Sigma}^{\prime}\left(t^{j}, \zeta^{j l}\right)$ aux sommets $t^{j}$ de $\Sigma$, dans des directions $\zeta^{j l}$ (telles que $\left.\left.t^{j}+\zeta^{j l} \in|\Sigma|\right), 1 \leqslant l \leqslant r(j), 1 \leqslant j \leqslant n+1\right)$ convenablement choisies.

Alors $\sigma$ sera (encore) déterminée de manière unique si l'on remplace la donnée de ses valeurs aux points $t \in \Sigma_{k} \backslash \Sigma^{0}$ par celle de ses dérivées premières $\sigma^{\prime}\left(t^{j} ; \zeta^{j l}\right), 1 \leqslant l \leqslant r(j), 1 \leqslant j \leqslant n+1$.

EXEMPLE : 4.2.1.

$$
k=3, n=2
$$

On remplace la donnée de $\sigma\left(\frac{2 t^{j}+t^{l}}{3}\right)$ par celle de $\sigma^{\prime}\left(t^{j} ; t^{l}-t^{j}\right), j, l=1,2,3, j \neq l$.

On peut supposer que $\sigma\left(\frac{t^{1}+t^{2}+t^{3}}{3}\right)$ est donné ou non. Dans les deux cas, $\sigma \in C^{0}(|\mathscr{C}|)$.

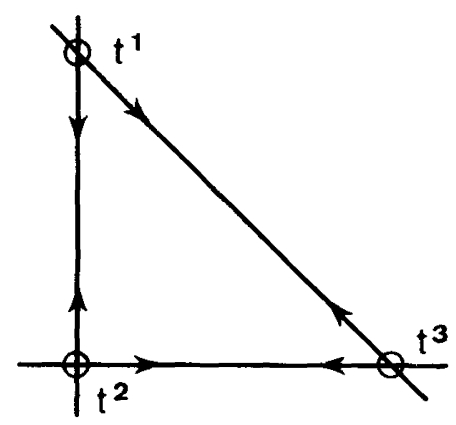

Figure 4.2.1

b) Dérivées d'ordre supérieur à 1. Éléments finis de classe $C^{r}, r \in \mathbf{N}^{*}$

\section{EXEMPLE 4.2.2.}

$$
k=4, n=2
$$

$P_{\Sigma}$ est déterminé de manière unique par la donnée simultanée de ses valeurs aux sommets de $\Sigma$ et de dérivées premières $P_{\Sigma}^{\prime}\left(t^{j} ; t^{l}-t^{t}\right)$

$$
\begin{array}{r}
P_{\Sigma}^{\prime}\left(\frac{t^{j}+t^{l}}{2} ; t^{q}-\frac{t^{j}+t^{l}}{2}\right), j, q, l=1,2,3, \\
j \neq l \neq q \neq j .
\end{array}
$$

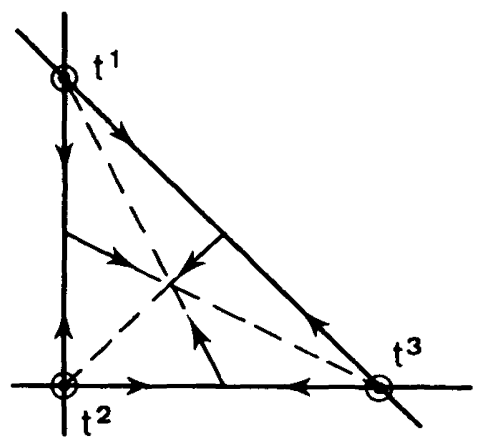

Figure 4.2.2

$\mathrm{n}^{\circ}$ août $1975, \mathrm{R}-2$. 
$\sigma$ sera (encore) déterminé de manière unique si l'on remplace la donnée de ses valeurs aux points de $\Sigma_{k} \backslash\left\{t^{1}, t^{2}, t^{3}\right\}$ par celle de ses dérivées premières $\sigma^{\prime}\left(t^{j} ; t^{j}-t^{l}\right), \sigma^{\prime}\left(\frac{t^{j}+t^{l}}{2} ; t^{q}-\frac{t^{j}+t^{l}}{2}\right), j, q, l=1,2,3, j \neq l \neq q \neq j$.

\section{EXEMPLE 4.2.3.}

$$
k=5, n=2
$$

On laisse au lecteur le soin de traiter de la même manière que dans l'exemple précédent, le cas où l'on se donne la valeur de $\sigma$, de ses différentielles premières et secondes aux sommets de $\Sigma$, et de ses dérivées premières

$$
\begin{array}{r}
\sigma^{\prime}\left(\frac{t^{j}+t^{l}}{2} ; t^{q}-\frac{t^{j}+t^{l}}{2}\right), j, l, q=1,2,3, \\
j \neq l \neq q \neq j .
\end{array}
$$

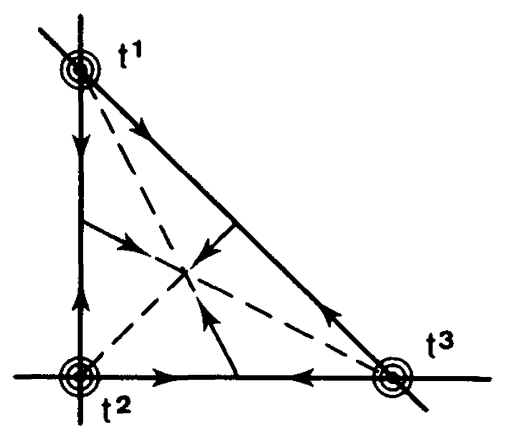

Figure 4.2.3

\section{BIBLIOGRAPHIE}

[1] AtreIA M., Fonctions " spline " et noyaux d'Aronszajn-Bergman, R.I.R.O., $\underline{n}^{\circ}$ 3, 1970.

[2] Bergman-Schiffer, Kernel functions and differential equations, Academic Press, 1953.

[3] Ciarlet P. G. and Raviart P. A., General Lagrange and Hermite interpolation in $R^{n}$ with applications to finite elements methods, Arch. Rat. Mech. Anal., 46 (1972), pp. 177-199. Interpolation Theory over Curved Element with applications to finite elements methods, Comp. Meth. Appl. Mech. Eng., 1 (1972), pp. 217-249.

[4] GARNIR, Analyse fonctionnelle, tome 1, Birkhauser-Verlag.

[5] GrotheNDIECK, Produits tensoriels topologiques, A.M.S.

[6] HiLton and WyLIE, Homology theory, Cambridge University Press.

[7] KaruIn S., Total Positivity, Standford, 1968.

[8] NeVEU J., Fonctions aléatoires gaussiennes, Cours de Montréal, 1968.

[9] NiCOLAIDES R. A., On a class of finite elements generated by Lagrande interpolation.

[10] Raviart P. A., Cours de D.E.A. sur les éléments finis, année 1971-1972.

[11] SCHWARTZ L., Sous-espaces hilbertiens d'espaces vectoriels tologiques et noyaux associés, J. d'Analyse Math., Jérusalem, 1964.

[12] Spanier E. H., Algebraic topology, Mac Graw Hill, 1966.

[13] Treves, Topological Vector Spaces, Distributions and Kernels, Academic Press, 1967.

[14] ZIENKIEwICZ, The finite element method, Mac Graw-Hill, 1971.

Revue Française d'Automatique, Informatique et Recherche Opérationnelle $\mathrm{n}^{\circ}$ août 1975, R-2. 